\title{
3 Research Square

\section{Influence of Genetic Background and Dietary Oleic Acid on Gut Microbiota Composition in Duroc and Iberian Pigs.}

Adrián López-García ( $\sim$ adrianlopgar@gmail.com )

Instituto Nacional de Investigación y Tecnología Agraria y Alimentaria https://orcid.org/0000-00028649-750X

Rita Benítez

INIA

Yolanda Núñez

INIA

Emilio Gómez-Izquierdo

ITACYL

Eduardo de Mercado

ITACYL

Juan María García Casco

INIA

Oscar González-Recio

INIA

Clemente López-Bote

UCM

Jordi Estellé

INRAE

Cristina Óvilo

INIA

\section{Research}

Keywords: gut microbiota, pig, oleic acid

Posted Date: December 23rd, 2020

DOI: https://doi.org/10.21203/rs.3.rs-131496/v1

License: (a) (i) This work is licensed under a Creative Commons Attribution 4.0 International License.

Read Full License 



\section{Influence of genetic background and dietary oleic acid on gut microbiota composition in Duroc and lberian pigs.}

Adrián López-García ${ }^{1 *}$, Rita Benítez ${ }^{1}$, Yolanda Núñez ${ }^{1}$, Emilio Gómez-Izquierdo ${ }^{2}$, Eduardo de Mercado $^{2}$, Juan M. García-Casco ${ }^{1}$, Óscar González-Recio ${ }^{1}$, Clemente López-Bote ${ }^{3}$, Jordi Estellé ${ }^{4}$ and Cristina Óvilo ${ }^{1}$

${ }^{1}$ Department of Animal Breeding, INIA. Madrid, Spain.

2 Pig Test Center ITACYL. Hontalbilla, Segovia, Spain.

3 Department of Animal Production, Veterinary Faculty, UCM. Madrid, Spain.

${ }^{4}$ GABI, INRAE, AgroParisTech, Université Paris-Saclay. Jouy-en-Josas, France.

\begin{tabular}{llll}
\hline Author's full name & e-mail address & Institution & Corresponding author \\
\hline Adrián López-García & $\underline{\text { adrian.lopez@inia.es }}$ & INIA & YES \\
Rita Benítez & $\underline{\text { rmbenitez@inia.es }}$ & INIA & \\
Yolanda Núñez & $\underline{\text { nunez.yolanda@inia.es }}$ & INIA & \\
Emilio Gómez-Izquierdo & gomizqem@itacyl.es & ITACYL & \\
Eduardo de Mercado & $\underline{\text { eduardo.mercado@inia.es }}$ & ITACYL & \\
Juan María García-Casco & garcia.juan@inia.es & INIA & \\
Óscar González-Recio & gonzalez.oscar@inia.es & INIA & \\
Clemente López-Bote & $\underline{\text { clemente@vet.ucm.es }}$ & UCM & \\
Jordi Estellé & jordi.estelle@inrae.fr & INRAE & \\
Cristina Óvilo & ovilo@inia.es & INIA & \\
\hline
\end{tabular}




\section{Abstract}

Background: Phenotypic variability for productive and meat quality traits has been largely studied in Iberian pigs, especially in genetic selection and nutritional experiments. Complex interactions among genetic background, diet composition and gut microbiota difficult the evaluation of true effects of each factor on phenotypes. In order to disentangle these interactions, we evaluated changes in gut microbiota composition comparing Iberian and Duroc pigs fed diets with different energy source.

Results: Gut microbiota composition was analysed in feces from 48 pigs (Iberian and Duroc) fed a standard diet or a sunflower oil-enriched diet with high oleic acid content (6\%). A higher richness was observed for Iberian pigs ( $\mathrm{p}<0.05$ ) and compositional analysis was applied for beta-diversity, differential abundance and pairwise log-ratio analyses. We found significant differences in overall microbiota composition between breeds, and also between diets inside breeds, to a lesser extent. Differential abundance analysis revealed that Duroc animals have more proportion of Actinobacteria and Prevotella, while Iberian replace those microorganisms with other more variable taxa. According to dietary differences, high-oleic fed animals were richer in Prevotella. We also found microbial ratios capable of separating animals by breeds and diets, mostly related to Actinobacteria.

Conclusion: This study reveals that both genetic background and diet composition might have a relevant impact in gut microbiota composition. The application of compositional data analysis has facilitated the identification of microorganisms and ratios as possibly related to metabolic changes due to genetic background and, to a lower extent, to dietary changes. This may lead to a relevant progress in the knowledge of interactions between pig genetics, environment and gut microbiota.

Keywords: gut microbiota; pig; oleic acid

\section{$1 \quad$ Background}

2 The contribution of Spanish pig production to worldwide pork market is meaningful. According to FAOSTAT, Spain

3 produces almost $4 \%$ of worldwide swine meat, being the fourth producer, and the second one inside EU, with a $19 \%$ of communal production, after Germany. One of the most eye-catching contribution of Spanish pig production to

5 international markets comes from Iberian pig, a local breed whose high-quality final products are much appreciated. In

6 fact, Spain ranks as the third country in lard and ham exportations (data from 2018) ${ }^{1}$, with a crucial contribution of dry-

7 cured Iberian ham. The high value of this product is due to the unique organoleptic properties conferred by Iberian pig

8 genetic, phenotypic and production particularities. This rustic and low-prolific local breed has been traditionally raised

9 under a semi-extensive production system at "dehesa" Mediterranean forests, taking advantage of the grass and acorn 
resources available ${ }^{2}$. The combination of their natural high tendency to fat accumulation and the acorn and grass feeding

11 in the late fattening phase ("Montanera"), confers their final products a unique fatty acid (FA) profile composition and a

12 higher intramuscular fat (IMF) content than commercial breeds.

13 The interest in this high-quality market has led to the search and legal regulation of strategies for the improvement of its 14 efficiency and productivity. In this sense, one of the most common practices nowadays is crossbreeding with Duroc, resulting in a negative impact on final product composition and quality ${ }^{3}$. Crossbreeding consequences have been largely studied through different points of view - such as phenotypic traits, meat quality, genetic markers or gene expression ${ }^{4-6}$. As the high quality of Iberian products has become the flag for the Iberian pig market to enter international markets, producers keep an interest in improving their products' organoleptic attributes through FA profile and IMF content optimization $^{7,8}$, especially in the recent more intensive production systems. Several strategies such as genetic selection, handling optimization and diet design, are open as key ways of modifying final product composition. Strategies such as dietary FA supplementation are common to enhance these organoleptic traits. Genetic, nutrition and nutrigenomic studies have shown the complexity of the molecular regulation of physiological processes associated to relevant phenotypic traits in Iberian pig genotypes subjected to different diets ${ }^{4,9-11}$

Besides, with the advent of new mass-sequencing techniques, alternative ways have been opened for animal breeding and for in-depth studies of the molecular basis of phenotypic variability. Microbiota studies have hugely benefitted from these NGS techniques, as culture-free sequencing allows obtaining a lot more information from microbiome communities. Gut microbiota has become a key focus in animal breeding industry, as well as in human health, since gut microbiota and host are linked in a bidirectional way; first, diet composition and other environmental elements, as well as animal genetics, might modify microbial population structure ${ }^{12}$; second, microbiota has been reported to have an impact on animal physiology, on multiple animal productive traits and also on the final products' quality and composition ${ }^{13,14}$. This intricate correlation network makes gut microbiota composition a key factor for understanding the relationships between genotype, phenotype and environment. As a consequence, it might result obvious that different genetic backgrounds can promote different microbiota population structures as it has been proven in other studies ${ }^{15}$. For this reason, the two main breeds employed in Iberian pig production (Iberian and Duroc) might have differences in gut microbiota composition possibly affecting relevant phenotypic traits. Also, dietary modification or supplementation strategies such as FA addition might have an impact on gut microbiota composition, potentially contributing to differences in animal traits, with special relevance on final product composition and quality. Understanding all these relationships and their implications is crucial to comprehend their global effect on animal phenotype.

In this study we compared the gut microbiota composition in pigs from Iberian and Duroc breeds, fed two different isocaloric and isoproteic diets with different energy source (oleic acid vs carbohydrates), in order to dissect the diet and 
breeds and diet groups, potentially mediated by gut microbiota.

\section{Methods}

\section{Experimental design and sampling}

The experiment was carried out at the facilities of the Instituto Tecnológico Agrario de Castilla y Leon (ITACYL) Pig Test Center (Hontalbilla, Segovia, Spain). A total of forty-eight castrated male purebred pigs, 19 Duroc (DU) and 29 Iberian (Torbiscal strain) ${ }^{16}$ (IB), were raised in the same commercial farm, weaned at 28 days old and transferred to the experimental facility one month after weaning. The animals were kept under identical management conditions, housed in batches of 4 pigs/pen $\left(1 \mathrm{~m}^{2} \mathrm{pig}^{-1}\right)$, with a concrete floor and straw bedding. Temperature was controlled at a mean of 23.8 ${ }^{\circ} \mathrm{C}$ throughout the experiment. At 70 days old ( \pm 2 days) and with a mean body weight of $19.7 \pm 3.6 \mathrm{~kg}(17.3 \pm 2.8 \mathrm{~kg} \mathrm{BW}$ in DU; $21.3 \pm 3.2 \mathrm{~kg} \mathrm{BW}$ in IB), both breed groups were distributed in two different experimental groups, with a factorial $2 \times 2$ design, and fed a control commercial diet with carbohydrates as energy source (C) (9 DU and 13 IB) or a sunflower oil-enriched diet with high oleic acid content $(6 \%)(\mathrm{O})(10 \mathrm{DU}$ and $16 \mathrm{IB})$. Both diets were formulated according to FEDNA nutritional guidelines (2006) using the Brill Formulation software (Brill Co., Georgia, USA) to be isocaloric and isoproteic (3.3 kcal digestible energy and 15.6\% crude protein) (Additional file 1) and were provided ad libitum. Diets were provided for 47 days, until animals reached $50.4 \pm 7.8 \mathrm{~kg} \mathrm{BW}(51 \pm 9 \mathrm{~kg} \mathrm{BW}$ in DU; $50 \pm 7 \mathrm{~kg} \mathrm{BW}$ in IB). Fresh water was provided ad libitum, with two drinking troughs available in each pen.

Animals were slaughtered at the end of treatment, at 117 days old ( \pm 2 days), and individual stool samples were then collected from rectum and rapidly frozen on liquid $\mathrm{N}_{2}$ and preserved at $-80^{\circ} \mathrm{C}$ until its use for microbiome analysis. All experiments were performed in accordance with the regulations of the Spanish Policy for Protection of Animals employed in Research and other scientific purposes RD53/2013, which meet the European Union Directive 2010/63/EU on the protection of animals used in experimentation. The project was approved on March 20, 2015, by the Comunidad de Madrid animal welfare and protection committee (reference number PROEX-007/15).

\section{DNA extraction and $16 S$ sequencing}

Microbiota DNA was extracted from the 48 stool samples using QIAamp PowerFecal® kit (QIAGEN, Hilden, Germany) according to manufacturer's standard protocol. Illumina MiSeq ${ }^{\circledR}$ paired-end sequencing protocol (Illumina, San Diego, CA, USA) was performed by an external service (FISABIO bioinformatics, Valencia, Spain) targeting 16S rRNA gene V3-V4 amplicon. The employed primers were S-D-Bact-0341-b-S-17 and S-D-Bact-0785-a-A-21 ${ }^{17}$, which produce amplicons of $464 \mathrm{bp}$. 
74 Raw reads were pre-processed using Prinseq-lite tool ${ }^{18}$ and in-house software. After trimming low quality bases in each read, sequences shorter than 50 bp and with an average quality score below 30 on a window of 20 bases were discarded. After joining forward and reverse files, a total amount of 11,599,350 reads were processed using Quantitative Insights Into Microbial Ecology (QIIME) version 1.9.1 ${ }^{19}$ into operational taxonomic units (OTUs), following the de novo OTUpicking approach, with a clustering threshold of 97\% identity. Chimeras were filtered using USEARCH algorithm (v. 6.1) 20. SILVA reference database (release 132) ${ }^{21}$ was used for taxonomic assignment and chimera filtering. An OTU abundance threshold of $0.005 \%$ was established for final quality-filtering, as described in Bokulich et al. ${ }^{22}$. Processed OTU table was composed by $9,428,777$ reads from 1,398 OTUs.

Finally, two additional processing steps were performed: a taxonomy filtering, excluding OTUs not assigned to Bacteria at kingdom level in the taxonomic classification, as used primers are designed for bacterial amplicons; and a sample pruning, removing those with sequencing depth lower than 30k total reads, which eliminated one sample for the IB-O group. The final OTU table was composed by 9,162,809 reads from 1,246 OTUs and 47 samples.

\section{Microbial community analysis}

All analyses were performed in R, using phyloseq package ${ }^{23}$. Microbiota composition was compared between breed-diet animal pens. Three different approaches were then followed to analyse microbiome composition: Alpha-diversity: For this approach, OTU table was rarefied to minimum sample depth (30,136 reads). Four alphadiversity measures were calculated: observed richness, Chao1 index, Shannon index and Inverse-Simpson index, using the function estimate_richness from phyloseq. ANOVA for each $\alpha$-diversity measure was carried out following this model:

$$
\alpha_{i j k}=\mu+b_{i}+d_{j}+b d_{i j}+e_{i j k}
$$

Being $\alpha$ the $\alpha$-diversity index for each sample, $b$ the Breed effect (with $i=2$ levels), $d$ the Diet effect (with $j=2$ levels) and $b d$ the interaction of both effects.

Data transformation: Compositional data analysis: Most of the statistical analysis were made taking into account the compositional nature of microbiome data ${ }^{24}$. For the compositional approach our non-rarefied dataset was processed following a number of steps as defined by ${ }^{25}$ : (1) Zero-counts imputing by Geometric Bayesian Multiplicative replacement; (2) Data closure computed to the total counts per sample; (3) Weighted centred log-ratio (CLR) transformation. This process moves the data to an additive scale, which makes the performance of multivariate hypothesis testing easier. The analysis was repeated glomming the dataset to OTU, genus, family and phylum levels. R packages zCompositions ${ }^{26}$ and easyCODA ${ }^{27}$ were used for this transformation. 

transformation of the data). For a better visualization of the behaviour of both breed and diet effects, a mixed "BreedDiet" factor was considered as PCA grouping variable. The distance matrix was built using Aitchison distance measure, which is the Euclidean distance after CLR transformation. Individual beta-diversities were calculated as the distance to centroid for groups from mixed "Breed-Diet" effect (using vegan::betadisper). Homogeneity of dispersions respect to the group centroids was measured through ANOVA-like permutation test (vegan::permutest) and post-hoc Tukey HSD test.

For testing the significance of multivariate effects in our a priori groups, non-parametric permutational multivariate analysis of variance (PERMANOVA) ${ }^{28,29}$ was performed using adonis2 function from vegan package in $\mathrm{R}^{30}$ with this model:

$$
D_{i j}=\mu+b_{i}+d_{j}+b d_{i j}+e_{i j}
$$

114 The additive model applied was built sequentially adding Breed $\left(b_{i} ; i=[1,2]\right)$ and Diet $\left(d_{j} ; j=[1,2]\right)$ factors and their interactions $\left(b d_{i j}\right)$. The Aitchison distance matrix $(D)$ was used as dependent variable.

\section{Differential abundance analysis}

118 Differential abundance analysis was carried out using both DESeq2 ${ }^{31}$ and ALDEx2 ${ }^{32} \mathrm{R}$ packages. Default DESeq2 normalization by estimation of size factors as the median ratio of counts ${ }^{33}$ and negative binomial GLM fitting with Wald significance tests were performed. Differences between breeds and between diets were analysed in separate models correcting by the other factor. Interactions between factors were also evaluated with an additional model (Table 1). Pvalues were corrected for multiple testing through Benjamini-Hochberg method. Significantly differentially abundant (DA) OTUs were considered with a false discovery rate (FDR) cut-off of 0.05 and a fold-change (FC) higher than 1.5 or lower than -1.5 (i.e., $|\log 2 \mathrm{FC}|>0.59)$.

Table 1: DESeq2 differential abundance models for each contrast, with $R$ notation.

\begin{tabular}{ll}
\hline Contrast & Model design \\
\hline Breed contrast & RA ratio $\sim$ Diet + Breed \\
Diet contrast & RA ratio $\sim$ Breed + Diet \\
Interaction effects & RA ratio $\sim$ Breed + Diet + Breed: Diet \\
\hline
\end{tabular}

127 Input for ALDEx2 analysis was prepared as the CLR-transformed posterior distribution of the data generated by 128 128 Monte Carlo samples from the Dirichlet distribution. CLR transformation was made through geometric mean of all 129 features' abundance. Mono-factorial breed and diet contrasts were performed, using Welch's t-test. P-values were 130 corrected through Benjamini-Hochberg method. OTUs were considered as significantly differentially abundant between

131 breeds or diets when corrected p-value was lower than 0.05 and diff.btw (i.e., median difference in CLR values between 132 factor groups) was higher than 1 or lower than -1. Visualization of coincident DA OTUs between contrasts and pipelines 133 was carried out with VennDiagram package in $\mathrm{R}^{34}$. 


\section{Pairwise log-ratios analysis}

136 Closured data were also used for another approach, using pairwise log-ratios ( $\mathrm{p}$-LR) instead of centred $\log$-ratios ${ }^{27}$, as a way to evaluate individual relationships among OTUs and features glommed to genus, family and phylum levels.

138 Recursive Partitioning and Regression Trees (RPART) were generated, including the microorganism pairwise log-ratios most suitable to split samples according to the Breed and Diet phenotypic criteria.

140 Pairwise log-ratios analysis is computationally expensive, as feature combinations produce matrices much larger than the 141 original, i.e., a $m \times n$ matrix (samples $\times$ features) would be transformed in a $m \times n(n-1) / 2$ matrix. For this reason, 142 OTU-grouped dataset was size-reduced by selecting a relevant feature subcomposition generated by sparse Partial Least 143 Square Discriminant Analysis (sPLS-DA), using the 4-leveled mixed trait "Breed-Diet" as classification factor. Five-fold 144 cross-validation with permutation was used to test the optimal component structure for the model. In order to generate the subcomposition, OTUs with a Variable Influence of Projection (VIP) value higher than 1 for each component were selected. As p-LR analysis keeps subcompositional coherence ${ }^{27}$, data subsetting has no significant effect on the results.

\section{Microbiome-phenotype association}

CLR data were also used to detect associations between microbiome and several phenotypic traits. IMF content was measured in Biceps femoris and Longissimus dorsi. FA profile was analysed in backfat outer layer (Back) and in ham outer fat layer (Ham). Total FA fractions (saturated, SFA; mono-unsaturated, MUFA; and poly-unsaturated, PUFA) as well as relevant FA contents (oleic, C18:1(9); linoleic, C18:2; palmitic, C16:0; and stearic, C18:0) were also measured, with all quantification methods being described in formerly published studies ${ }^{11}$.

PERMANOVA controlling both Breed and Diet effects was performed for each phenotypic variable using vegan::adonis2 function, in order to elucidate linear or nonlinear relationships between samples distribution and each phenotypic trait:

$$
D_{i j}=\mu+b_{i}+d_{j}+P+e_{i j}
$$

157 The additive model applied was built sequentially adding Breed $\left(b_{i} ; i=[1,2]\right)$ and Diet $\left(d_{j} ; j=[1,2]\right)$ factors, and each 158 phenotype $(P)$ was treated as linear regression at the end. The Aitchison distance matrix $(D)$ was used as dependent 159 variable.

160 Additionally, individual OTU association was addressed using Limma linear regression. To avoid the influence of the

161 known breed effects on phenotype which could lead to potential spurious associations, both microbiome and phenotypic datasets were split for carrying out independent DU and IB microbiome-phenotype analyses. To measure diet weight in correlations, two methods were carried out: (1) linear regression of each OTU on each phenotypic variable without diet correction; (2) linear regression of each OTU on the diet regression residuals of each phenotypic variable. P-values were adjusted using Benjamini-Hochberg method (adjusted significance threshold set to FDR $=0.05$ ). Representation of 
associations between OTUs and phenotypes was addressed through interaction network in Cytoscape software (v. 3.8.1),

setting Kamada-Kawai algorithm (Edge-weighted spring embedded layout) ${ }^{35}$ for optimal visualization.

168

\section{Results}

Overall microbiota composition

171 The overall composition is summarised in Table 2. Our final OTU table was composed by 47 samples, as we pruned one sample with less than 30k reads. As mentioned in methods, we applied an additional filter removing those reads not assigned to Bacteria kingdom. After this step, $97.44 \%$ of total reads were kept, representing 1,246 OTUs. From this bacteria-classified fraction, $99.93 \%$ reads were assigned to phylum level. A total of 17 phyla were identified. Prevalence and abundance of phyla are represented in Figure 1.

Table 2: Overall microbiota composition.

\begin{tabular}{|c|c|c|c|c|c|c|}
\hline & Kingdom & Phylum & Class & Order & Family & Genus \\
\hline$\%$ Sequences $^{1}$ & 97.44 & 99.93 & 99.91 & 99.91 & 97.30 & 86.70 \\
\hline Classified OTUs & 1246 & 1245 & 1243 & 1243 & 1184 & 988 \\
\hline Identified taxa & 1 & 17 & 23 & 33 & 48 & 148 \\
\hline
\end{tabular}

Total OTU number, $\%$ of sequences assigned to a known taxon at different ranks and unique taxa found at each rank.

${ }^{1}$ Proportion of total reads classified to phylum and lower taxonomic levels are calculated relative to the total reads after filtering OTUs not assigned to Bacteria kingdom.

177 The 988 annotated OTUs were classified to 148 different genera, covering $86.7 \%$ of total reads. 20 genera had an average 178 relative abundance (RA) higher than 1\%, being Prevotella_9, Lactobacillus, Prevotellaceae NK3B31 group,

179 Alloprevotella and Treponema_2 the most abundant ones (Additional file 2). Note that SILVA database subdivides some 180 genera according to sequence clustering, appearing under multiple designations in our database.

181 On the other hand, 79 genera were present at an average abundance lower than $0.1 \%$ (Additional file 2). This fact might 182 be taken into account when interpreting the results, as they might be poorly represented across the samples (i.e., low 183 prevalence) and/or have a minimum number of reads per sample (i.e., low coverage). Nonetheless, all the analyses have been performed including these low-abundance OTUs.

Microbiota composition analysis:

Two-way ANOVA for $\alpha$-diversity measures in rarefied data showed no significant differences between diet groups (C vs O), but all of them (richness: $p=0.021$; Chao1 index: $p=0.018$; Shannon index: $p=0.008$; and Inverse Simpson index: $\mathrm{p}=0.002)$ were significantly affected by breed, these measures showing higher values in the IB group (Figure 2). Initial average number of OTUs per sample was 1,046 for DU population and 1,097 for IB population. After rarefaction, these numbers were corrected to richness values, with an average of 880 in DU and 944 in IB. through PC1. Diet clustering can be observed through PC2, but only when features are analysed at lower taxonomic levels 

between diets. At OTU level, centroid distance is maximum for both breed and diet groups. Individual beta-diversities were calculated as the Euclidean distance to centroids for groups from mixed "Breed-Diet" effect. Average individual dispersion between groups was not significantly different, except for OTU-glommed dataset. At OTU level, DU-C samples had an average beta-diversity index significantly lower compared to DU-O, IB-C and IB-O groups, although pvalue in DU-C vs IB-C contrast (same diet in different breeds) was close to 0.05 . (Figure 4).

PERMANOVA analysis confirmed that samples from different breeds are significantly different. Differences between diets (corrected by breed effect) are also significant at OTU, genus and family level, although these differences blur at phylum level (Table 3). Nonetheless, the differences between groups are moderate, as roughly $10 \%$ of variance from our data can be explained by Breed effect, and only $5 \%$ by Diet effect.

Table 3: PERMANOVA statistics.

\begin{tabular}{cllll} 
& & F statistic & $\mathbf{R}^{\mathbf{2}}$ & p-value \\
\hline \multirow{4}{*}{ Phylum } & Breed & 5.05 & 0.10 & $0.0017^{*}$ \\
& Diet & 1.44 & 0.03 & 0.1776 \\
& Interaction & 1.32 & 0.03 & 0.2187 \\
\hline \multirow{4}{*}{ Family } & Breed & 5.96 & 0.11 & $0.0001^{*}$ \\
& Diet & 2.24 & 0.04 & $0.0283^{*}$ \\
& Interaction & 1.51 & 0.03 & 0.1247 \\
\hline \multirow{3}{*}{ Genus } & Breed & 6.15 & 0.12 & $0.0001^{*}$ \\
& Diet & 2.55 & 0.05 & $0.0118^{*}$ \\
& Interaction & 1.27 & 0.02 & 0.1859 \\
\hline \multirow{3}{*}{ OTU } & Breed & 5.39 & 0.10 & $0.0001^{*}$ \\
& Diet & 3.43 & 0.07 & $0.0021^{*}$ \\
& Interaction & 1.17 & 0.02 & 0.2313 \\
\hline
\end{tabular}

F statistic, $\mathrm{R}^{2}$ and $\mathrm{p}$-values at different taxonomic levels.* $\mathrm{p}<0.05$.

205

\section{Differential abundance (DA) analysis}

Two separate contrasts were made, for breed and diet factors, using an FDR cut-off of 0.05 and a fold-change minimum threshold of $\pm 1.5\left(\log _{2} F C \approx \pm 0.59\right)$ for DESeq2 analysis, and a minimum median difference between CLR values of $\pm 1($ diff. $b t w= \pm 1)$ for ALDEx2 analysis.

Figure 5 includes volcano plots for both contrasts with DESeq2 and ALDEx2 methods. Both breed and diet contrasts resulted in more restrictive results with ALDEx2 method. Breed contrast shows a higher number of over-abundant (OA) OTUs in IB pigs when done with DESeq2, while ALDEx2 breed contrast shows a higher number of DU OA OTUs. On the other hand, O diet presents a higher number of OA OTUs than under-abundant (UA) OTUs, compared to C diet, both with DESeq2 and ALDEx2. With DESeq2 pipeline, 468 DA OTUs from 119 different genera were found for DU vs IB contrast, mostly from Ruminococcaceae, Prevotellaceae and Lachnospiraceae families. On the other hand, 185 DA OTUs from 71 genera were found between $\mathrm{C}$ and $\mathrm{O}$ diet groups, predominated by the same families. With ALDEx2, 207 DA OTUs were found for breed contrast, whereas only 18 DA OTUs were found for diet test, mostly from Prevotellaceae 
family. Common DA OTUs between pipelines are counted in Table 4. For breed contrast, the most representative taxa in overlapping DA OTUs was Prevotellaceae (mostly Prevotella) (27\%), followed by Lachnospiraceae (16\%), Ruminococcaceae (15\%) and Veillonellaceae (mostly Megasphaera and Anaerovibrio OTUs) (11\%). For diet contrast only 18 OTUs were common to both pipelines, 7 of them from Prevotellaceae family. Supplementary material with the complete DA-OTU list for all contrasts is also available (Additional file 3).

Table 4: DA OTUs by factor and pipeline.

\begin{tabular}{llrrrr}
\hline \multirow{2}{*}{ DESeq2 } & & DA OTUs & DA genera & DA families & DA phyla \\
\hline \multirow{2}{*}{ ALDEx2 } & Duroc vs Iberian & 468 & 119 & 44 & 13 \\
& Control vs High Oleic & 185 & 71 & 32 & 9 \\
\hline \multirow{2}{*}{ Overlap } & Duroc vs Iberian & 207 & 72 & 22 & 6 \\
& Control vs High Oleic & 18 & 13 & 8 & 3 \\
\hline & Duroc vs Iberian & 151 & 63 & 20 & 6 \\
& Control vs High Oleic & 18 & 13 & 8 & 3 \\
\hline
\end{tabular}

Number of DA OTUs in breed and diet contrasts using DESeq2 or ALDEx2 pipelines, and common DA OTUs between both pipelines. Number of genera, families and phyla grouping these OTUs are also shown.

We evaluated coincident DA OTUs among contrasts and methods, as shown in the Venn diagram (Figure 6). When comparing DESeq2 with ALDEx2, 151 DA OTUs were coincident in the breed contrast and 18 coincidences were found in the diet contrast. On the other hand, when comparing the results obtained for breed and diet effects within the same method, we detected some coincident OTUs (75 OTUs in DESeq2 and only 1 OTU in ALDEx2). Only one OTU is common to all four comparisons, the one named as denovo378129, from genus Shuttleworthia, which was OA in DU and

231 Significant interaction between breed and diet was detected for 26 OTUs $\left(\mathrm{P}_{\mathrm{adj}}<0.05\right)$, most of them being qualitative 232 interactions (Figure 7). Most of these OTUs were members of Corynebacterium, Lactobacillus and Ruminococcaceae 233 taxa.

Pairwise log-ratio analysis

sPLS-DA for OTU-grouped dataset confirmed a total of 141 relevant OTUs (VIP $\geq 1$ ) as predictive of the "Breed-Diet" 237 phenotype. This subcomposition has been used to calculate pairwise log-ratios at OTU level. For genus, family and 238 phylum grouped datasets, full table has been used for p-LR calculation. Recursive Partitioning and Regression Trees 239 (RPART) were built for each p-LR matrix, as shown in Figure 8. We observed that the best classification occurs at 240 family level, being the ratios Corynebacteriaceae/Spirochaetaceae (COR/SPR) and 241 Peptostreptococcaceae/Streptococcaceae (PEP/STR) the most relevant to classify between breeds.

242 At phylum level diet differences were not clear inside each breed, as it was observed in beta-diversity analysis. 243 Actinobacteria/Epsilonbacteraeota ratio was able to separate samples by breed, being reliable to classify as IB but not as 244 DU, and was not capable of properly separate diet groups inside each breed. 
At family level, three ratios were capable of separating animals in the four Breed-Diet groups with high precision. As mentioned, COR/SPR and PEP/STR ratios mainly clustered Iberian and Duroc separately, but also differentiated dietary groups inside the IB breed, while Atopobiaceae/Enterobacteriaceae (ATO/ENT) ratio separated diets within the DU animals. These ratios are closely related with those responsible of group classification at genus level (Anaerostipes/Corynebacterium_1 and Family_Peptostreptococcaceae/Peptococcus), except that these genera ratios can only discriminate between IB-C, IB-O and DU animals, but no ratio was able to differentiate diets within DU breed.

In the case of COR/SPR, both ratio components were responsible of variation. ALDEx2 DA analysis at family level showed that both Corynebacteriaceae and Spirochaetaceae were significantly DA between DU and IB groups (IB-UA: diff.btw $=-3.23$ and IB-OA: diff.btw $=1.13$, respectively) although Corynebacteriaceae reduction in IB is stronger than Spirochaetaceae increment. As Corynebacteriaceae are Actinobacteria, breed separation at phylum level is reflected at lower taxonomic levels. Same occurs at the genus decision tree, whose first ratio Anaerostipes/Corynebacterium_l was able to differentiate breeds, with Corynebacterium having the highest weight, as DA analysis at genus level stated a Corynebacterium_l OA in DU animals genus (diff.btw $=-2.78)$.

The PEP/STR ratio was able to identify IB animals, even separating $\mathrm{C}$ and $\mathrm{O}$ populations when accounting also COR/SPR ratio. DA analysis revealed that Peptostreptococcaceae family was more abundant in DU animals (diff.btw $=-1.27$ ), while Streptococcaceae family was not differentially abundant between breeds. At genus level, Peptococcus abundance played a similar role, as IB pigs had a higher RA (diff.btw $=1.14)$.

As indicated, ATO/ENT ratio was able to distinguish between C and O diets within DU animals, but only when applied after discriminating IB animals with former ratios (otherwise it would only discriminate between DU-O and the rest of Breed-Diet groups). Enterobacteriaceae were OA in DU animals (diff.btw $=-1.92)$ but no abundance differences were detected between diet groups.

Finally, the two representative ratios at OTU level (denovo219246/denovo273762 and denovo277739/denovo351253) were composed by OTUs assigned to Rickenellaceae/Ruminococcaceae families and Peptococcus/Lactobacillus genera, respectively. Additionally, denovo219246 and denovo277739 appeared as DA between IB and DU animals (first one with

\section{Association with phenotypic traits}

PERMANOVA did not show any significant association between overall microbiota composition and the analysed phenotypic traits. When the effect of each individual OTU was evaluated through Limma regression different results were 
a total of 87 individual OTUs were found as significantly associated to phenotypic traits in IB subset (Figure 9). Most of them belonged to Alloprevotella and other Prevotella groups and were associated to multiple traits. Ruminococcaceae OTUs were positively linked to oleic acid and negatively linked to palmitic acid proportions in backfat, while Rikenellaceae OTUs mainly appear negatively associated to oleic acid proportion, affecting in both cases overall MUFA and SFA proportions. Some relevant OTUs from Shuttleworthia, Lactobacillus and Corynebacterium genera also appeared associated to FA composition. When diet correction was applied almost every correlation disappeared, and only one OTU was negatively correlated to ham oleic acid proportion in IB subset, denovo37314 (Treponema 2).

In this study we explore the composition and disparities of the bacterial fraction of fecal microbiota populations between two different pig breeds (Iberian and Duroc) at 117 days old, fed diets with different energy source (carbohydrates vs. sunflower oil with high oleic acid content). Some important differences were detected between Iberian and Duroc populations, and to a lesser extent also between control and high-oleic diets.

Overall taxonomic structure revealed Bacteroidetes, Firmicutes and Proteobacteria as the most abundant phyla, with an important predominance of Prevotellaceae, Lachnospiraceae and Ruminococcaceae families. Prevotella genus was the most abundant, with an average RA of 30\% in Duroc animals and 22\% in Iberian animals when considering all SILVA genus-clustering designations. Prevotella are anaerobic saccharolytic bacteria which produce acetate and succinate through fermentation ${ }^{36}$ and their abundance is highly related to high-fiber long-term diets ${ }^{37,38}$. Their presence in fecal microbiota is highly reported in human ${ }^{39}$ and other animals such as ruminants, predominating in rumen microbiota ${ }^{40-42}$. Multiple studies have shown the dominance of Prevotella in pig gut microbiota ${ }^{43}$ as well as their relevance in enterotypes ${ }^{44}$ or even immune response ${ }^{45}$. Alloprevotella genus is also one of the most representative in our data, with an average RA of $4.2 \%$ in DU and $11.6 \%$ in IB animals. This genus is also known for its saccharolytic activity ${ }^{46}$, although it is less studied than Prevotella due to its lower abundance in gut communities. Lachnospiraceae and Ruminococcaceae families also comprehend well-known polysaccharide fermenters, which in addition participate in methanogenesis by producing $\mathrm{H}_{2}$ and formate as substrates to archaeal communities ${ }^{47}$. Abundance balance of these three families in gut microbiota, through a number of abundance ratios, might be relevant for host traits related with feed efficiency, metabolism or health. For instance, Prevotella-to-Bacteroides ratio has been related with weight and fat loss in humans ${ }^{48}$, and Firmicutes-toBacteroidetes ratio has been repeatedly associated to fiber metabolism or obesity in human populations ${ }^{37,49}$ and murine models ${ }^{50}$, although there is controversy about these links ${ }^{51}$. Prevotellaceae, Lachnospiraceae and Ruminococcaceae also compose the core microbiome in rumen, being shared by multiple ruminants in an important abundance ${ }^{52}$. Our data support that this core microbiota might also be shared by other monogastric species along all digestive tract. Similar findings are reported by Crespo-Piazuelo et al. ${ }^{53}$ when analysing gut microbiota in different intestinal regions using the 
same IB-C pig population. However, they reported a range of 35 to $41 \%$ of Prevotella abundance within all colon regions.

These discrepancies must be attributed to different intestinal region, different genotype and diet of the total animal

311 population studied (colon from IB-C animals vs rectum from IB and DU fed C and O diets).

312 Alpha-diversity measures revealed a higher number of different OTUs in IB pigs, which can also explain the higher values 313 of Shannon and Simpson indices. These interesting findings might be related with the higher rusticity and resilience of 314 Iberian pig breed compared with other commercial breeds ${ }^{54}$, since higher ecological diversity is associated with a better response to environmental disturbances (response diversity) ${ }^{55}$.

Beta-diversity PCA showed us that overall microbiome composition is different between DU and IB animals. Diet effects appear masked by this breed influence, but its impact on microbiome structure is also noticeable. Although no significant interaction between breeds and diets was detected with PERMANOVA, PCA showed a slightly different separation of C and $\mathrm{O}$ centroids within each breed, which could suggest a different response to diet between Duroc and Iberian microbial communities. On the other hand, PCA average dispersion within DU-C samples is lower compared to the rest of groups, further exploration. are pretty similar, and DU-O group has a higher dispersion within its samples, although no significant differences were detected with respect to IB groups. This higher dispersion level within DU-O cluster compared to DU-C is coincident with the increment in alpha-diversity variability detected for this group, especially when observing richness or Chao1 index values, and opposes to the sample homogeneity within DU-C. In contrast, IB-O animals did not show different alpha-diversity mean or dispersion values compared to IB-C. Thus, it can be hypothesised that Duroc animals might respond in a more heterogeneous way to dietary oleic acid increment.

Differential abundance analysis gives a more specific insight about these changes in microbiome population structure among experimental groups. DESeq2 is a RNAseq-based, robust and well documented method broadly used in microbiome DA analyses. However, in recent years the concern about compositional nature of these data has increased, and some authors remark that RNAseq-based tools are not accounting for the compositional nature of microbiome data. For this reason we also performed differential abundance analysis with ALDEx2 method, an ANOVA-based approach which accounts for this intrinsic compositionality and has a similar sensitivity than other methods, also reducing false positive rate near to zero ${ }^{24}$. The most conservative strategy would be to focus in the overlapping DA OTU set between DESeq2 and ALDEx2, but due to the restrictive behaviour of ALDEx2 we decided to consider also DESeq2 results for

Our results comparing diets ( $\mathrm{C}$ vs $\mathrm{O}$ ) showed 18 OTUs as DA with both DESeq2 and ALDEx2 methods (i.e. all the OTUs detected by ALDEx2 were included in the DESeq2 output). 12 of them were high-oleic OA OTUs from Prevotellaceae, Lachnospiraceae, Muribaculaceae and Ruminococcaceae families, while the remaining 6 UA OTUs were 3 Rikenellaceae members and 3 OTUs classified as Clostridium s.s. 6, Erysipelotrichaceae UCG-004 and Desulfovibrio, 
respectively. When exploring DESeq2 results, most of the high-oleic OA OTUs were from the same families, but a high number of OA Prevotella and Alloprevotella OTUs was detected. Within UA OTUs, a higher variety of families was present, with high representativity of Prevotellaceae, Ruminococcaceae, Rikenellaceae and Erysipelotrichaceae. The greater presence of Prevotella and kindred taxa in $\mathrm{O}$ animals might be related with the higher fiber content in this diet, as it was formulated to be isoenergetic in relation to control diet. Fiber content has already been linked to gut microbiota changes ${ }^{56}$, and Prevotella OTUs have been defined as biomarkers for high-fiber diets ${ }^{57}$. Generally speaking, high-oleic OA OTUs were rare OTUs (i.e., RA $\leq 0.01 \%$ in C group), while high-oleic UA OTUs tended to be more common (i.e., RA $>0.01 \%$ in $\mathrm{C}$ group), meaning that the abundance decrease of common OTUs due to oleic acid and fiber supplementation results in an abundance increment of marginal OTUs. This fact is supported by our Shannon index values showing an upward trend in $\mathrm{O}$ group compared to $\mathrm{C}$ diet, which, in this case, means a higher overall evenness in $\mathrm{O}$ group, as observed richness is not different between diets.

Breed differences were more obvious, matching with PCA sample distribution, as centroids were more distant between breeds than between diets. Based on the overlapped results from DESeq2 and ALDEx2, DU animals have a higher abundance of Actinobacteria, specifically from Corynebacterium and Bifidobacterium genera, as well as multiple Lachnospiraceae (Blautia, Roseburia, Lachnoclostridium, Fusicatenibacter, and Shuttleworthia), multiple Prevotellaceae OTUs (mostly Prevotella and Alloprevotella) and other genera such as Clostridium s.s. 1, Holdemanella, Megasphaera or Succinivibrio, while IB OA OTUs were scarcer and belonged to more diverse taxa (see Additional file 3 for complete OTU list). As far as we know, no former studies have found these differences between Duroc and Iberian pigs, so these findings might be important to define unique microbiome patterns associated to host genotypes. Still, gut microbiota contrasts between Duroc, Landrace and Large White pigs also revealed a higher presence of Prevotella in Duroc animals ${ }^{15}$.

Bifidobacterium are well known anaerobic lactic acid producer bacteria ${ }^{58}$ whose abundance in fecal microbiota has been reported as negatively correlated with pig age, being more abundant at early life stages ${ }^{59}$. The transition from weaning to adult cereal-based diet is a key factor in the replacement of these early-colonizers by other microorganisms such as Prevotella, Roseburia or Succinivibrio, more typical of adult fecal microbiota ${ }^{45}$. Only one Bifidobacterium OTU was detected in our data, presenting low abundance (mean $\mathrm{RA}=0.01 \%$ ) and majorly absent in IB animals. This might indicate a slower replacement of weaning to adult microbiota in DU pigs, which might as well be related to their lower alphadiversity. fermentative organisms with special relevance at amino acid biosynthesis pathways, such as lysine through intermediate diaminopimelate (DAP) from aspartate and pyruvate, or histidine ${ }^{60,61}$. Other authors reported lower protein synthesis, degradation and deposition rates in Iberian pigs, compared to other commercial breeds ${ }^{62}$. Corynebacterium might be 
contributing to this differential protein synthesis and degradation balance, through a differential bioavailability of essential amino acids in the gut, thus, their abundance could be correlated to animal protein deposition.

Differential abundance of other groups might be related with richness and resilience of Iberian pigs. In this sense, the lower abundance of multiple Prevotella OTUs in IB pigs seem to be related with the previously reported increase of microbial diversity, as it happened when comparing $\mathrm{C}$ and $\mathrm{O}$ diets. This richness increment might likewise be related with the lower abundance of Shigella-Escherichia group, as a higher $\alpha$-diversity has been associated to the prevention in the establishment of pathogenic microbes such as Enterobacteriaceae ${ }^{37}$. Treponema DA OTUs, which are virtually absent in Duroc animals, appear as moderately abundant in Iberian pigs $(\mathrm{RA} \approx 1 \%$ ), thus these fiber-fermenters might have settled the niche that Prevotella left. Finally, we detected an abundance opposition between Prevotella and Treponema taxa. The most abundant OTUs of all the dataset, belonging to Prevotella genus, were OA in DU, while several Treponema OTUs were OA in IB. As described by Crespo-Piazuelo et al. ${ }^{53}$, this might suggest a competition for dietary fibre. Finally, denovo378129 (Shuttleworthia) was the only OTU differentially abundant between all breed and diet groups. This bacterial group is relatively unknown, although it has been reported as a typical member of animal gut microbiome in several studies ${ }^{59,63}$. According to our results, Shuttleworthia could be a key differentiator between Iberian and Duroc animals, having a relevant link either with fiber or with oleic acid dietary supply.

Additionally, DESeq2 allowed us to test interaction effects between breed and diet factors. With this method we were able to find as much as 26 significant interactions, most of them being qualitative. According to DESeq2 DA analysis for diets (C vs O) using DU and IB subpopulations, we were able to classify these interactions based on the different response to diets inside each breed: no response to $\mathrm{O} \operatorname{diet}(\mathrm{NR})$, abundance increase due to $\mathrm{O} \operatorname{diet}(\mathrm{AI})$ or abundance decrease due to $\mathrm{O} \operatorname{diet}(\mathrm{AD}) .12$ OTUs were NR within DU animals (7 IB-AI and 5 IB-AD) while 7 OTUs were NR within IB animals (all of them DU-AI). Only 1 OTU (denovo347841) presented an abundance increase in response to O diet within both breeds, being its AI much larger for Iberian animals (quantitative interaction). The remaining 6 interaction-significant OTUs were not significantly DA between diets within each breed. The OTU denovo347841 has been classified as Unclassified_Muribaculaceae, a relatively unknown Bacteroidales family which has been observed in multiple gut microbiomes through $16 \mathrm{~S}$ or metagenomic analyses ${ }^{64}$. Although their main metabolic role remains unknown, their phylogenetic closeness to Prevotellaceae and Bacteroidaceae could reveal saccharolytic activity. In summary, most of the observed interactions are qualitative interactions in which one of the breeds responds to the diet while the other one does not. Also, no pure qualitative interactions are observed showing opposite responses to the diet in both breeds. The presence of interactions for individual OTU abundances would be a clear indicator of differential effects of diet on the gut microbiota of Duroc and Iberian pigs. This fact can be related with our beta-diversity results, as our PCA showed a tendency to a higher centroid distance within IB-C and IB-O groups than within DU-C and DU-O, although the absence of significant interactions in PERMANOVA test throws some uncertainty about this conclusion. Also, former studies 
carried out with the same animals have proven the existence of a differential response to $\mathrm{O}$ diet for several productive traits and for adipose tissue transcriptome ${ }^{11}$. In summary, this differential response may exist for gut microbiome composition and structure, but again might be not clearly noticed due to the low number of animals and to the relatively high resemblance between feed formulations and to the intrinsic difficulty in evaluating and interpreting interaction effects.

410 We also used the log-ratio analysis approach, assuming that ratios between OTUs can also be biologically relevant despite 411 of single association with OTU RAs. Thus, composition differences have been explored more deeply by means of p-LR analysis, which detected those microorganism pairwise ratios whose abundances could separate animals by both breed and diet. In order to check which microbes were mainly responsible of separation between groups at each ratio, supplementary differential abundance analyses were made, using ALDEx2 (as this pipeline uses the same methods as for RPART trees building) with phylum, family and genus-grouped database. With this approach we identified some ratios that could be useful as differentiators between Duroc and Iberian phenotypes. Actinobacteria abundance has been observed to be important when compared to Epsilonbacteraeota (Phyl. Nov.), a novel phylum proposed by Waite et al. ${ }^{65}$ as a separate group from former Epsilonproteobacteria class, which was classified inside Proteobacteria phylum. Our dataset contained Campylobacter and Helicobacter members of Epsilonbacteraeota, two genera with diverse metabolisms more known by their pathogenic species affecting human and other animals. One out of our OTUs has been classified as Campylobacter hyointestinalis, a species name proposed to Campylobacter isolated from pigs with proliferative enteritis ${ }^{66}$. Interaction relationship of these pathogenic bacteria and Actinobacteria (Corynebacterium, Bifidobacterium, Collinsella, Enterorhabdus and non-classified Atopobiaceae) present in our database remains unknown. However, as Epsilonbacteraeota RAs remain fairly constant across samples, differentiation between IB and DU pigs might be due to lower Actinobacteria abundances in Iberian animals. Actinobacteria relevance in breed separation has been upheld when analysing ratios at lower taxonomic levels. Accordingly, Corynebacteriaceae/Spirochaetaceae and Anaerostipes/Corynebacterium were relevant in breed separation at family and genus levels, respectively. In any case, complete separation between breeds and diets, including the discrimination between Duroc diet groups, is only possible at family level making use of three pairwise ratios (COR/SPR, PEP/STR and ATO/ ENT), considering that Enterobacteriaceae are more abundant in DU-O group. This fact also reflects the masking effect that breed is causing to differentiate diet effects on microbial populations, previously commented. This type of approach might be interesting to detect microbial biomarkers, given their importance as tools for genetic diagnosis or diet determination. In the case of Iberian pig industry this might be especially useful in order to address frauds due to crossbreeding and dietary manipulation, which can importantly affect final products' quality ${ }^{6,7}$. 
As an additional approach we related the individual OTU abundances with different fat composition traits, in order to deepen in the potential effects of the gut bacteria on host's metabolism. A former study with the same animals reported significant differences in FA composition between $\mathrm{C}$ and $\mathrm{O}$ diets ${ }^{67}$. Our first Limma approach (breed subsetting and no diet correction) was used to globally visualize the correlations between OTU abundances and lipidic composition, independently of their causal relationship. Again, differences were detected between DU and IB, as only one OTU abundance was significantly correlated to FA composition in DU animals. When the second Limma approach was applied (breed subsetting and diet correction) we saw that most of these correlations were caused by the diet influence. In IB subpopulation, Prevotellaceae OTUs mostly appear negatively correlated to SFA and/or positively correlated to MUFA (as the variation in one FA alters the proportion of the others). As they have a role in fiber digestion, these correlations might be caused by the higher fiber content in high-oleic diet, as mentioned before. Other correlations might be directly caused by increased supply of dietary oleic acid, such as the negative correlations between OTUs from Lactobacillus (denovo2335, denovo274149) and backfat SFA content, or between Campylobacter (denovo141328) and both ham and backfat SFA content. Lactobacillus are important fermenters with a crucial role in VFA biosynthesis ${ }^{68}$, so its association with FA availability could be expected. Regarding Campylobacter, as it happens with C. jejuni, C. hyointestinalis might also lack common microbial metabolic pathways related to carbohydrate utilization, replacing them by a rapid utilization of amino acids and SCFA such as lactate and acetate ${ }^{69}$. Thus, its correlation with FA composition might be related to differential SCFA and amino acid bioavailability between dietary groups. Only one OTU was detected as correlated with FA after correcting by diet (second Limma approach), denovo37314 (Treponema 2), negatively correlated with ham oleic acid content in IB. This suggests that direct (i.e., not caused by diet differences) association between gut microbiota and FA muscle accretion might also exist. Unfortunately, our experiment does not allow to detect these associations, as intragroup variability is very low for lipidic traits and sample size does not provide enough statistical power. Further experimental designs standardizing breed and diet might be adequate to focus on causal relationships between gut microbiota composition and host lipidic traits.

As our experiment has focused on the bacterial subset of fecal microbiota, interactions with other microbial clades, especially eukaryotes, must be studied for a better understanding of the real nature of microbial population changes. On the other hand, metabolite variations between breeds and diets must be analyzed in order to stablish the true relationship between microbiome and host phenotype. Mid-gut microbiota populations might also be interesting to observe dietary responses, as multiple digestion processes occur at superior gut sections. Finally, the complexity in analysing crossover the differences found in this study, although interaction between effects must not be ignored.

\section{Conclusions}


Our study reveals that genetic background has an important impact on pig gut microbiota composition, while dietary

changes have a samller and more variable effect, which could be highly dependent on fiber content. It also brings light to the complexity of microbial relationships. We report a higher OTU richness in Iberian pigs, which highlights the importance of genetic background (i.e., animal breed) in overall microbiome composition resemblance, and may suggest a potential relationship between gut microbiota diversity and composition and Iberian pigs' resilience. Dietary modifications had a small effect in microbiome composition, much less important than host genotype. Differential abundance revealed a complex picture of microbe relationships, with a considerable effect of the breed and multiple interactions between breed and diet. In spite of this complexity, we identified relevant DA taxa and taxa ratios as potentially associated to the metabolic differences between breeds and to a lower extent for the diet influence. Finally, an approach to the use of OTU pairwise ratios as a predictive tool has been performed, with some interesting results that should be subject of further researches.

\section{Acknowledgements}

The authors are grateful to the staff of the "Pig Test Center" ITACYL (Hontalbilla, Segovia, Spain) for technical assistance with husbandry and sampling. We also thank GABI-INRA for hosting the research stay for the development of bioinformatic pipelines.

\section{Funding}

This research was funded by the Comunidad de Madrid (Spain) and European Structural and Investment Funds (project MEDGANCM S2013/ABI-2913), and the Spanish Ministry of Science and Innovation (grant PID2019-108695RB-C31). A.L.G. was funded by FPI-INIA grant with reference FPI-SGIT2016-06.

\section{Abbreviations}

AD: Abundance decrease

AI: Abundance increase

ATO/ENT: Atopobiaceae/Enterobacteriaceae

BW: Body weight

CLR: Centred log-ratio

COR/SPR: Corynebacteriaceae/Spirochaetaceae

DA: Differential abundance

FA: Fatty acid

FC: Fold-change

FDR: False discovery rate

IMF: Intramuscular fat

MUFA: Mono-unsaturated fatty acids

NR: no response

OA: Over-abundant

OTU: Operational taxonomic unit

p-LR: Pairwise log-ratio

PCA: Principal Component Analysis

PERMANOVA: Permutational multivariate analysis of variance

PEP/STR: Peptostreptococcaceae/Streptococcaceae

PUFA: Poly-unsaturated fatty acids

RA: Relative abundance

RPART: Recursive Partitioning and Regression Trees

SFA: Saturated fatty acids

SCFA: Sort-chained fatty acids

SPLS-DA: sparse Partial Least Square Discriminant Analysis

UA: Under-abundant

VIP: Variable Influence of Projection 


\section{Ethics approval and consent to participate}

All experiments were performed in accordance with the regulations of the Spanish Policy for Protection of Animals employed in Research and other scientific purposes RD53/2013, which meet the European Union Directive 2010/63/EU on the protection of animals used in experimentation. The project was approved on March 20, 2015, by the Comunidad de Madrid animal welfare and protection committee (reference number PROEX-007/15).

\section{Competing interests}

The authors declare that they have no competing interests.

\section{Consent for publication}

Not applicable.

\section{Author information \\ Affiliations}

Department of Animal Breeding, INIA. Madrid, Spain.

Adrián López-García, Rita Benítez, Yolanda Núñez, Juan M. García-Casco, Óscar González-Recio, Cristina Óvilo.

Pig Test Center ITACYL. Hontalbilla, Segovia, Spain.

Emilio Gómez-Izquierdo, Eduardo de Mercado.

Department of Animal Production, Veterinary Faculty, UCM. Madrid, Spain.

Clemente López-Bote.

GABI, INRAE, AgroParisTech, Université Paris-Saclay. Jouy-en-Josas, France.

Jordi Estellé.

\section{Contributions}

C.Ó. and C.L.B. obtained funding and conceived the experiments. C.Ó., J.G.C. and E.G.I. designed the experiments. R.B., Y.N., E.D.M., E.G.I. and A.L.G. performed the experiments. A.L.G. analysed the data. A.L.G., O.G.R. and J.E. contributed to bioinformatics analysis. A.L.G. and C.Ó. wrote the paper. All authors revised and approved the submitted paper.

\section{References}

1. FAO. FAOSTAT Database. Food and Agriculture Organization of the United Nations. http://www.fao.org/faostat/es/\#data/TP/visualize. Published 2020. Accessed August 17, 2020.

2. López-Bote CJ. Sustained utilization of the Iberian pig breed. Meat Science. 1998;49:17-27. doi:10.1016/S0309-1740(98)90036-5

3. Ventanas S, Ventanas J, Ruiz J. Sensory characteristics of Iberian dry-cured loins: Influence of crossbreeding and rearing system. Meat Science. 2007;75(2):211-219. doi:10.1016/j.meatsci.2006.07.003

4. Óvilo C, Benítez R, Fernández AI, et al. Longissimus dorsi transcriptome analysis of purebred and crossbred Iberian pigs differing in muscle characteristics. BMC Genomics. 2014;15(1):413. doi:10.1186/1471-2164-15-413

5. Robina A, Viguera J, Perez-Palacios T, et al. Carcass and meat quality traits of Iberian pigs as affected by sex and crossbreeding with different Duroc genetic lines. Spanish Journal of Agricultural Research. 2013;11(4):1057-1067. doi:10.5424/sjar/2013114-4637

6. Muñoz M, García-Casco JM, Alves E, et al. Development of a 64 SNV panel for breed authentication in Iberian pigs and their derived meat products. Meat Science. 2020;167:108152. doi:10.1016/j.meatsci.2020.108152

7. Daza A, Olivares A, Rey AI, Ruiz J, López-Bote CJ. Iberian pig production: The problems of success. Options Méditerranénnes. 2008;A(78):163-171. http://om.ciheam.org/om/pdf/a78/00800259.pdf. Accessed October 28, 2020.

8. Wood JD, Enser M, Fisher A v., et al. Fat deposition, fatty acid composition and meat quality: A review. Meat Science. $2008 ; 78(4): 343-358$. doi:10.1016/j.meatsci.2007.07.019

9. Ayuso M, Fernández AI, Isabel B, et al. Long term vitamin A restriction improves meat quality parameters and modifies gene expression in Iberian pigs. Journal of Animal Science. 2015;93(6):2730-2744. doi:10.2527/jas.2014-8573

10. Ayuso M, Fernández AI, Núñez Y, et al. Developmental stage, muscle and genetic type modify muscle transcriptome in pigs: Effects on gene expression and regulatory factors involved in growth and metabolism. PLOS ONE. 2016;11(12):167858. doi:10.1371/journal.pone.0167858

11. Benítez R, Trakooljul N, Núñez Y, et al. Breed, diet, and interaction effects on adipose tissue transcriptome in iberian and duroc pigs fed different energy sources. Genes. 2019;10(8):589. doi:10.3390/genes10080589

12. Hanning I, Diaz-Sanchez S. The functionality of the gastrointestinal microbiome in non-human animals. Microbiome. 2015;3:51. doi:10.1186/s40168-015-0113-6

13. Khanal P, Maltecca C, Schwab C, Fix J, Tiezzi F. Microbiability of meat quality and carcass composition traits in swine. 2020. doi:10.21203/rs.3.rs-22906/v1 
14. Verschuren LMG, Calus MPL, Jansman AJM, et al. Fecal microbial composition associated with variation in feed efficiency in pigs depends on diet and sex. Journal of Animal Science. 2018;96(4):1405-1418. doi:10.1093/jas/sky060

15. Bergamaschi M, Tiezzi F, Howard J, et al. Gut microbiome composition differences among breeds impact feed efficiency in swine. Microbiome. 2020;8(1):110. doi:10.1186/s40168-020-00888-9

16. Rodríguez-Valdovinos C, García-Casco J, García-Ruiz F, Núñez-Moreno Y, Silió-López L. A further look of the genetic origin and singularity of the Torbiscal Iberian pig line. Spanish Journal of Agricultural Research. 2019;17(1):1-13. doi:10.5424/sjar/2019171-13618

17. Klindworth A, Pruesse E, Schweer T, et al. Evaluation of general 16S ribosomal RNA gene PCR primers for classical and next-generation sequencing-based diversity studies. Nucleic Acids Research. 2013;41(1):e1-e1. doi:10.1093/nar/gks808

18. Schmieder R, Edwards R. Quality control and preprocessing of metagenomic datasets. Bioinformatics. 2011;27(6):863-864. doi:10.1093/bioinformatics/btr026

19. Caporaso JG, Kuczynski J, Stombaugh J, et al. QIIME allows analysis of high-throughput community sequencing data. Nature Methods. 2010;7(5):335-336. doi:10.1038/nmeth.f.303

20. Edgar RC. Search and clustering orders of magnitude faster than BLAST. Bioinformatics. 2010;26(19):2460-2461. doi:10.1093/bioinformatics/btq461

21. Quast C, Pruesse E, Yilmaz P, et al. The SILVA ribosomal RNA gene database project: Improved data processing and web-based tools. Nucleic Acids Research. 2013;41(D1):D590-6. doi:10.1093/nar/gks1219

22. Bokulich NA, Subramanian S, Faith JJ, et al. Quality-filtering vastly improves diversity estimates from Illumina amplicon sequencing. Nature Methods. 2013;10(1):57-59. doi:10.1038/nmeth.2276

23. McMurdie PJ, Holmes SP. phyloseq: An R Package for Reproducible Interactive Analysis and Graphics of Microbiome Census Data. PLoS ONE. 2013;8(4):e61217. doi:10.1371/journal.pone.0061217

24. Gloor GB, Macklaim JM, Pawlowsky-Glahn V, Egozcue JJ. Microbiome datasets are compositional: And this is not optional. Frontiers in Microbiology. 2017;8(NOV):2224. doi:10.3389/fmicb.2017.02224

25. Greenacre M, Lewi P. Distributional equivalence and subcompositional coherence in the analysis of compositional data, contingency tables and ratio-scale measurements. Journal of Classification. 2009;26(1):29-54. doi:10.1007/s00357-009-9027-y

26. Palarea-Albaladejo J, Martín-Fernández JA. ZCompositions - R package for multivariate imputation of left-censored data under a compositional approach. Chemometrics and Intelligent Laboratory Systems. 2015;143:85-96. doi:10.1016/j.chemolab.2015.02.019

Greenacre M. Compositional Data Analysis in Practice. New York: Chapman and Hall/CRC; 2018. doi:10.1201/9780429455537

McArdle BH, Anderson MJ. Fitting multivariate models to community data: A comment on distance-based redundancy analysis. Ecology. 2001;82(1):290-297. doi:10.1890/0012-9658(2001)082[0290:FMMTCD]2.0.CO;2

Anderson MJ. A new method for non-parametric multivariate analysis of variance. Austral Ecology. 2001;26(1):32-46. doi:https://doi.org/10.1111/j.1442-9993.2001.01070.pp.X

30. Oksanen J, Blanchet FG, Friendly M, et al. Vegan: community ecology package. 2018. doi:ISBN 0-387-95457-0

31. Love MI, Huber W, Anders S. Moderated estimation of fold change and dispersion for RNA-seq data with DESeq2. Genome Biology. 2014;15(12):550. doi:10.1186/s13059-014-0550-8

32. Fernandes AD, Reid JNS, Macklaim JM, McMurrough TA, Edgell DR, Gloor GB. Unifying the analysis of high-throughput sequencing datasets: Characterizing RNA-seq, 16S rRNA gene sequencing and selective growth experiments by compositional data analysis. Microbiome. 2014;2(1):15. doi:10.1186/2049-2618-2-15

33. Anders S, Huber W. Differential expression analysis for sequence count data. Genome Biology. 2010;11(10):R106. doi:10.1186/gb-2010-11$10-\mathrm{r} 106$

34. Chen H, Boutros PC. VennDiagram: A package for the generation of highly-customizable Venn and Euler diagrams in R. BMC Bioinformatics. 2011;12(1):35. doi:10.1186/1471-2105-12-35

35. Kamada T, Kawai S. An algorithm for drawing general undirected graphs. Information Processing Letters. 1989;31(1):7-15. doi:10.1016/00200190(89)90102-6

36. Shah HN, Collins DM. Prevotella, a new genus to include Bacteroides melaninogenicus and related species formerly classified in the genus Bacteroides. International Journal of Systematic Bacteriology. 1990;40(2):205-208. doi:10.1099/00207713-40-2-205

37. de Filippo C, Cavalieri D, di Paola M, et al. Impact of diet in shaping gut microbiota revealed by a comparative study in children from Europe and rural Africa. Proceedings of the National Academy of Sciences of the United States of America. 2010;107(33):14691-14696. doi:10.1073/pnas.1005963107

38. Chen T, Long W, Zhang C, Liu S, Zhao L, Hamaker BR. Fiber-utilizing capacity varies in Prevotella- versus Bacteroides-dominated gut microbiota. Scientific Reports. 2017;7(1). doi:10.1038/s41598-017-02995-4

39. Arumugam M, Raes J, Pelletier E, et al. Enterotypes of the human gut microbiome. Nature. 2011;473(7346):174-180. doi:10.1038/nature09944

40. Li J, Zhong H, Ramayo-Caldas Y, et al. A catalog of microbial genes from the bovine rumen reveals the determinants of herbivory. bioRxiv. 2018:272690. doi:10.1101/272690

41. Lee HJ, Jung JY, Oh YK, Lee SS, Madsen EL, Jeon CO. Comparative survey of rumen microbial communities and metabolites across one caprine and three bovine groups, using bar-coded pyrosequencing and 1H nuclear magnetic resonance spectroscopy. Applied and Environmental Microbiology. 2012;78(17):5983-5993. doi:10.1128/AEM.00104-12

42. Lopes LD, de Souza Lima AO, Taketani RG, et al. Exploring the sheep rumen microbiome for carbohydrate-active enzymes. Antonie van Leeuwenhoek, International Journal of General and Molecular Microbiology. 2015;108(1):15-30. doi:10.1007/s10482-015-0459-6

43. Xiao L, Estellé J, Kiilerich P, et al. A reference gene catalogue of the pig gut microbiome. Nature Microbiology. 2016;1(12):1-6. doi:10.1038/nmicrobiol.2016.161

44. Ramayo-Caldas Y, Mach N, Lepage P, et al. Phylogenetic network analysis applied to pig gut microbiota identifies an ecosystem structure linked with growth traits. ISME Journal. 2016;10(12):2973-2977. doi:10.1038/ismej.2016.77

45. Mach N, Berri M, Estellé J, et al. Early-life establishment of the swine gut microbiome and impact on host phenotypes. Environmental Microbiology Reports. 2015;7(3):554-569. doi:10.1111/1758-2229.12285

46. Downes J, Dewhirst FE, Tanner ACR, Wade WG. Description of Alloprevotella rava gen. nov., sp. nov., isolated from the human oral cavity, and reclassification of Prevotella tannerae Moore et al. 1994 as Alloprevotella tannerae gen. nov., comb. nov. International Journal of Systematic and Evolutionary Microbiology. 2013;63(PART4):1214-1218. doi:10.1099/ijs.0.041376-0

47. Seshadri R, Leahy SC, Attwood GT, et al. Cultivation and sequencing of rumen microbiome members from the Hungate1000 Collection. Nature Biotechnology. 2018;36(4):359-367. doi:10.1038/nbt.4110 
48. Hjorth MF, Blædel T, Bendtsen LQ, et al. Prevotella-to-Bacteroides ratio predicts body weight and fat loss success on 24-week diets varying in macronutrient composition and dietary fiber: results from a post-hoc analysis. International Journal of Obesity. 2019;43(1):149-157. doi:10.1038/s41366-018-0093-2

49. Xu P, Li M, Zhang J, Zhang T. Correlation of intestinal microbiota with overweight and obesity in Kazakh school children. BMC Microbiology. 2012;12. doi:10.1186/1471-2180-12-283

50. Ley RE, Bäckhed F, Turnbaugh P, Lozupone CA, Knight RD, Gordon JI. Obesity alters gut microbial ecology. Proceedings of the National Academy of Sciences of the United States of America. 2005;102(31):11070-11075. doi:10.1073/pnas.0504978102

51. Magne F, Gotteland M, Gauthier L, et al. The firmicutes/bacteroidetes ratio: A relevant marker of gut dysbiosis in obese patients? Nutrients. 2020;12(5). doi:10.3390/nu12051474

52. Wallace RJ, Sasson G, Garnsworthy PC, et al. A heritable subset of the core rumen microbiome dictates dairy cow productivity and emissions. Science Advances. 2019;5(7). doi:10.1126/sciadv.aav8391

53. Crespo-Piazuelo D, Estellé J, Revilla M, et al. Characterization of bacterial microbiota compositions along the intestinal tract in pigs and their interactions and functions. Scientific Reports. 2018;8(1). doi:10.1038/s41598-018-30932-6

54. Forero Vizcaíno FJ, Andrés AI. El Cerdo Ibérico Una Revisión Transversal. (Forero Vizcaíno FJ, ed.). Sevilla: Consejería de Agricultura y Pesca (Andalucía); 2008.

55. Elmqvist T, Folke C, Nystrom M, et al. Response Diversity, Ecosystem Change, and Resilience. Frontiers in Ecology and the Environment 2003;1(9):488. doi: $10.2307 / 3868116$

56. Heinritz SN, Weiss E, Eklund M, et al. Intestinal microbiota and microbial metabolites are changed in a pig model fed a high-fat/low-fiber or a low-fat/high-fiber diet. PLOS ONE. 2016;11(4):154329. doi:10.1371/journal.pone.0154329

57. le Sciellour M, Labussière E, Zemb O, Renaudeau D. Effect of dietary fiber content on nutrient digestibility and fecal microbiota composition in growing-finishing pigs. PLoS ONE. 2018;13(10). doi:10.1371/journal.pone.0206159

58. Pokusaeva K, Fitzgerald GF, van Sinderen D. Carbohydrate metabolism in Bifidobacteria. Genes and Nutrition. 2011;6(3):285-306. doi:10.1007/s12263-010-0206-6

59. Han GG, Lee JY, Jin GD, et al. Tracing of the fecal microbiota of commercial pigs at five growth stages from birth to shipment. Scientific Reports. 2018;8(1):6012. doi:10.1038/s41598-018-24508-7

60. Liu Y, White RH, Whitman WB. Methanococci Use the Diaminopimelate Aminotransferase (DapL) Pathway for Lysine Biosynthesis. Journal of Bacteriology. 2010;192(13):3304-3310. doi:10.1128/JB.00172-10

61. Oliveira A, Oliveira LC, Aburjaile F, et al. Insight of genus Corynebacterium: Ascertaining the role of pathogenic and non-pathogenic species. Frontiers in Microbiology. 2017;8(OCT):1937. doi:10.3389/fmicb.2017.01937

62. Rivera-Ferre MG, Aguilera JF, Nieto R. Differences in whole-body protein turnover between Iberian and Landrace pigs fed adequate or lysinedeficient diets. In: Journal of Animal Science. Vol 84. Oxford Academic; 2006:3346-3355. doi:10.2527/jas.2005-405

63. Wallace RJ, Rooke JA, McKain N, et al. The rumen microbial metagenome associated with high methane production in cattle. BMC Genomics. 2015;16(1):839. doi:10.1186/s12864-015-2032-0

64. Lagkouvardos I, Lesker TR, Hitch TCA, et al. Sequence and cultivation study of Muribaculaceae reveals novel species, host preference, and functional potential of this yet undescribed family. Microbiome. 2019;7(1):28. doi:10.1186/s40168-019-0637-2

65. Waite DW, Vanwonterghem I, Rinke C, et al. Comparative genomic analysis of the class Epsilonproteobacteria and proposed reclassification to epsilonbacteraeota (phyl. nov.). Frontiers in Microbiology. 2017;8(APR). doi:10.3389/fmicb.2017.00682

66. Gebhart CJ, Edmonds P, Ward GE, Kurtz HJ, Brenner DJ. "Campylobacter hyointestinalis" sp. nov.: a new species of Campylobacter found in the intestines of pigs and other animals. Journal of Clinical Microbiology. 1985;21(5):715-720. doi:10.1128/jcm.21.5.715-720.1985

67. Benítez R, Fernández A, Isabel B, et al. Modulatory effects of breed, feeding status, and diet on adipogenic, lipogenic, and lipolytic gene expression in growing iberian and duroc pigs. International Journal of Molecular Sciences. 2018;19(1). doi:10.3390/ijms19010022

68. Hammes WP, Vogel RF. The genus Lactobacillus. In: The Genera of Lactic Acid Bacteria. Springer US; 1995:19-54. doi:10.1007/978-1-46155817-0_3

69. Stahl M, Butcher J, Stintzi A. Nutrient acquisition and metabolism by Campylobacter jejuni. Frontiers in cellular and infection microbiology. 2012;2:5. doi:10.3389/fcimb.2012.00005

\section{Figures}

Figure 1 - Phyla prevalence and abundance in dataset. Each dot represents an OTU, being the $\mathrm{x}$-axis its average relative abundance and the y-axis the proportion of samples in which it is present. "Unclassified_Bacteria" is the denomination given to those OTUs with no phylum assignment.

Figure $2-\alpha$-diversity boxplots according to Breed (left) and Diet (right) groups in rarefied data.

Figure $3-\beta$-diversity plot from PCA of CLR-transformed data, at phylum, family, genus and OTU levels and variance percentage explained by each component. Ellipses show the Normal-theory confidence regions with alpha $=0.95$. Centroids are represented by diamond-shaped points.

Figure $4-\beta$-diversity indices by group at different feature glom levels. Tukey HSD test group letters and p-values for significant group comparisons $(\mathrm{p}<0.05)$ are represented.

Figure 5 - Volcano plots of differentially abundant OTUs using DESeq2 (A, B) and ALDEx2 (C, D), for breed (left) and diet (right) contrasts. Red dots represent OTUs with an adjusted p-value below the FDR cut-off $(0.05)$ and a FC value above 1.5 or below -1.5 for DESeq 2 contrasts, or a median difference between CLR values above 1 or below -1 for ALDEx 2 contrasts. OTUs with $\log 2(\mathrm{FC})<0$ or diff.btw $<0$ are more abundant in Duroc and Control groups, respectively.

Figure 6 - Venn Diagram comparing DA OTUs in Breed (red) and Diet (blue) contrasts using both DESeq2 and ALDEx2.

Figure 7 - Interaction plots for each OTU with significant interaction between breed and diet factors for DA analysis with DESeq2. Normalized counts from DESeq2 algorithm and standard error of the mean (SEM) are represented in y-axis. 
Figure 8 - RPART trees from pairwise log-ratios at phylum, family, genus and OTU levels. Each decision level indicates the threshold value of one p-LR to classify a sample in one phenotypic group or another. Each node box displays the classification, the probability of each class at that node (i.e. the probability of the class conditioned on the node) and the percentage of observations used at that node. Class probabilities at each node are sorted as: DU-C, DU-O, IB-C and IB-O.

Figure 9 - Network representing individual OTU significant correlation with each phenotypic trait within IB subpopulation, from Limma regression analysis, when no diet correction was applied. Edge colour: red = positive correlation; blue = negative correlation. Central nodes have more connections (i.e., significantly associated to more phenotypic traits) than peripheral nodes. Numbered nodes represent OTUs classified as genera with high relevance due to their abundance patterns in our dataset.

\section{Supplementary information}

Additional file 1 - Diet composition.

Nutrient and fatty acid composition for Control (C) and High-Oleic (O) diets.

\section{Additional file 2 - Relative abundance of genera}

Tables containing overall relative abundance of identified genera and relative abundance by Breed-Diet groups. Mean and standard deviation of RA are shown for each OTU, as well as taxonomic classification. RA_Rank column represents the rareness of each OTU (High_RA: RA $\geq 1 \%$; Mid_RA: $1 \%>$ RA $\geq 0.1 \%$; Low_RA: RA $<0.1 \%$ ).

Additional file 3 - Differentially abundant OTUs

Tables containing differentially abundant OTUs for each factor (Breed and Diet) with both pipelines (DESeq2 and ALDEx2) and overlapping of both pipelines. Prevalence represents the proportion of samples in which each OTU is found. For DESeq2, normalized counts per group (Ncounts), fold change (log2FC) and adjusted p-value (padj) are shown. For ALDEx2, relative abundance per group (RA), median difference in CLR values between factor groups (diff.btw) and Welch's test adjusted p-value (we.eBH) are shown. In overlapping tables information of both pipelines is shown. 


\section{Figures}

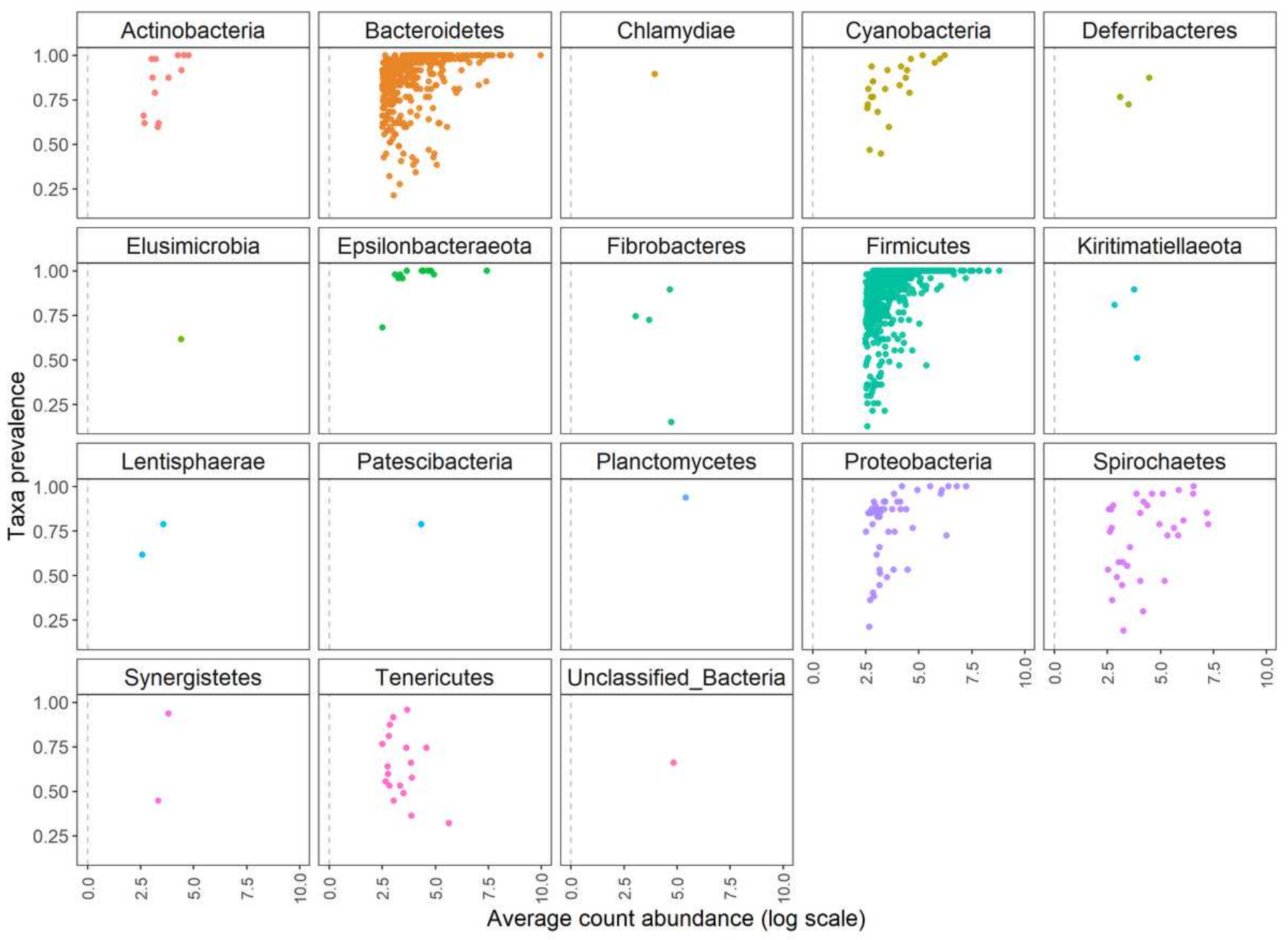

\section{Figure 1}

Phyla prevalence and abundance in dataset. Each dot represents an OTU, being the $x$-axis its average relative abundance and the y-axis the proportion of samples in which it is present. "Unclassified_Bacteria" is the denomination given to those OTUs with no phylum assignment. 

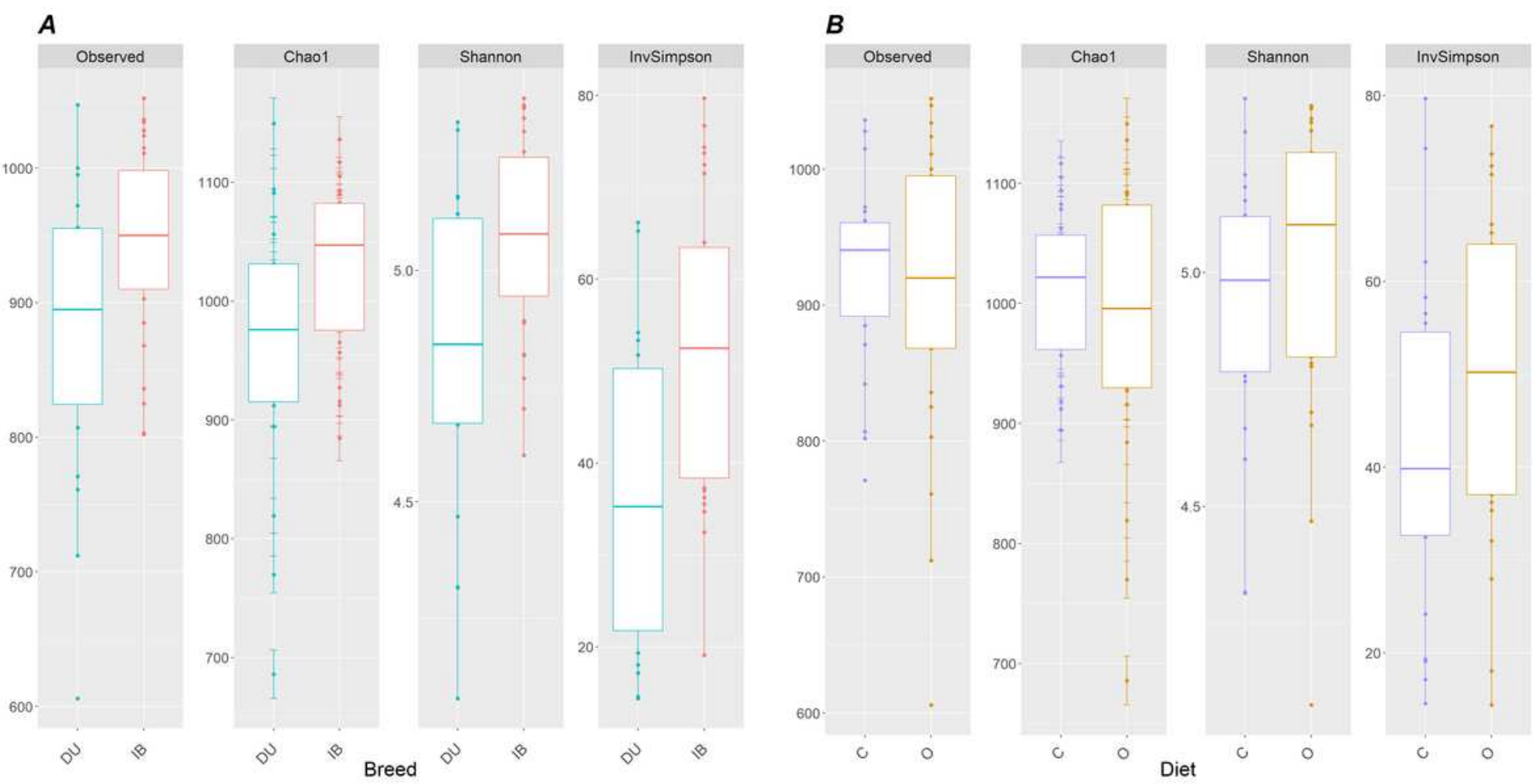

Figure 2

a-diversity boxplots according to Breed (left) and Diet (right) groups in rarefied data. 

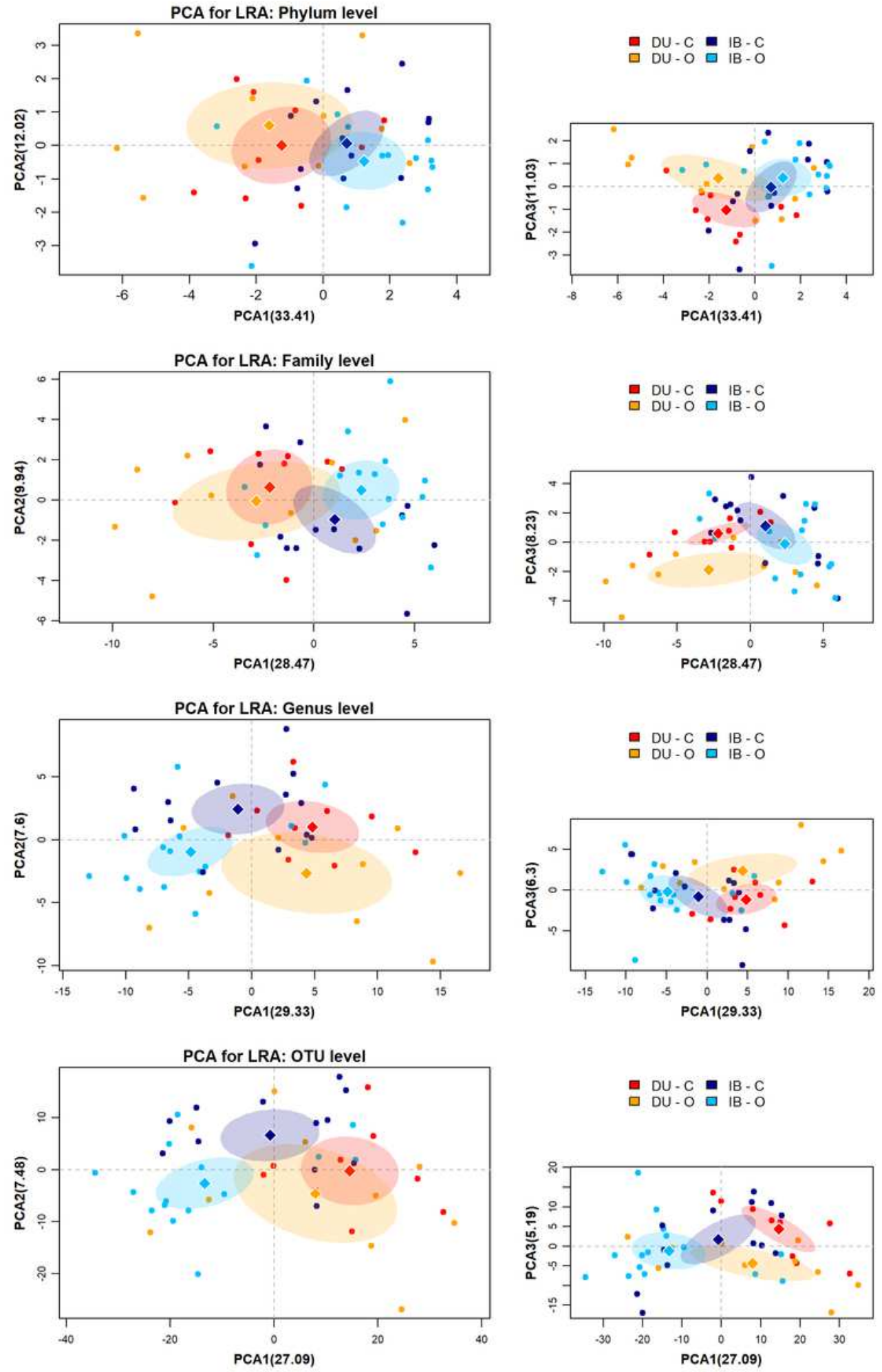

\section{Figure 3}

$\beta$-diversity plot from PCA of CLR-transformed data, at phylum, family, genus and OTU levels and variance percentage explained by each component. Ellipses show the Normal-theory confidence regions with alpha $=0.95$. Centroids are represented by diamond-shaped points. 


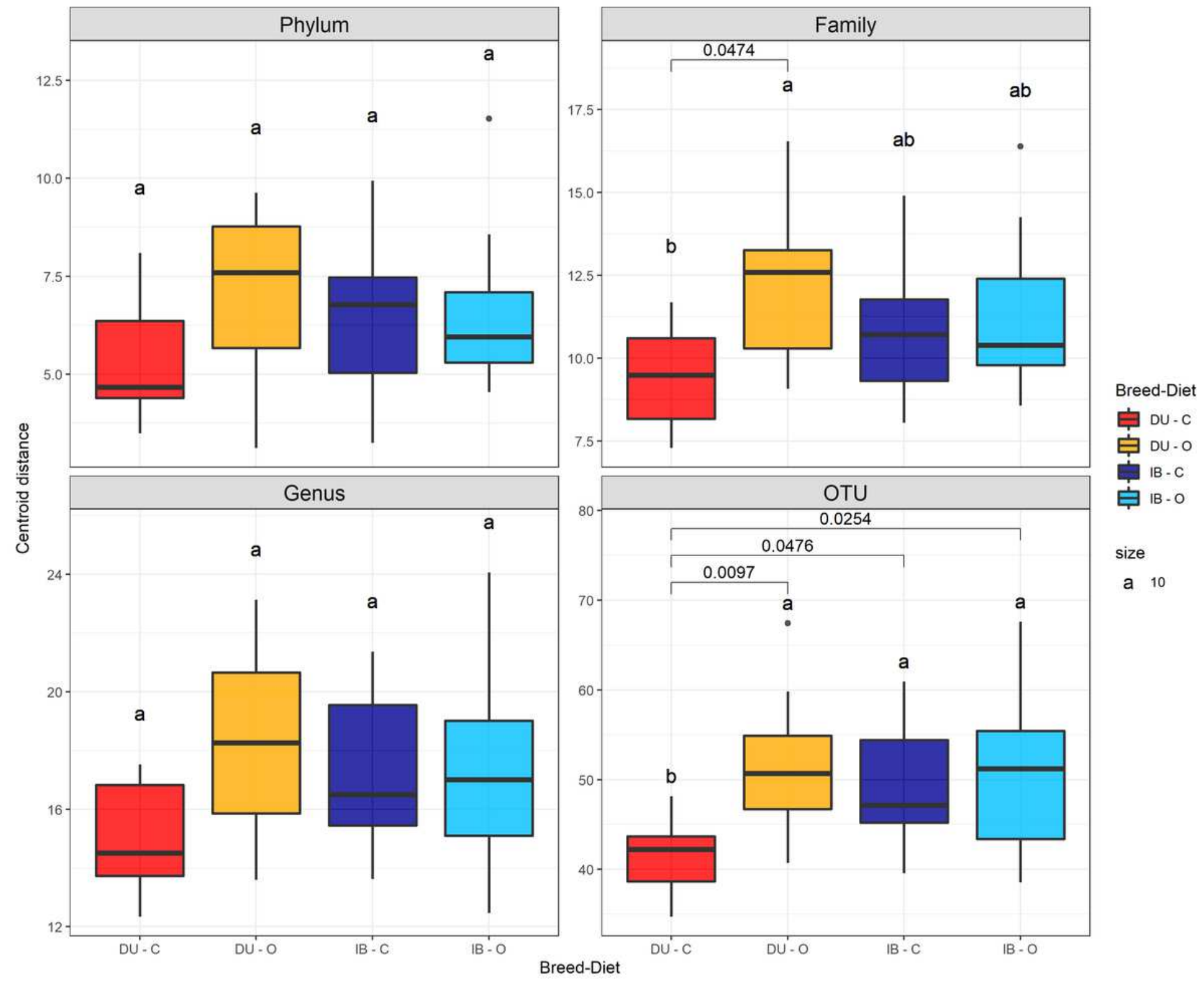

Figure 4

$\beta$-diversity indices by group at different feature glom levels. Tukey HSD test group letters and $p$-values for significant group comparisons $(p<0.05)$ are represented. 
A Duroc vs lberian

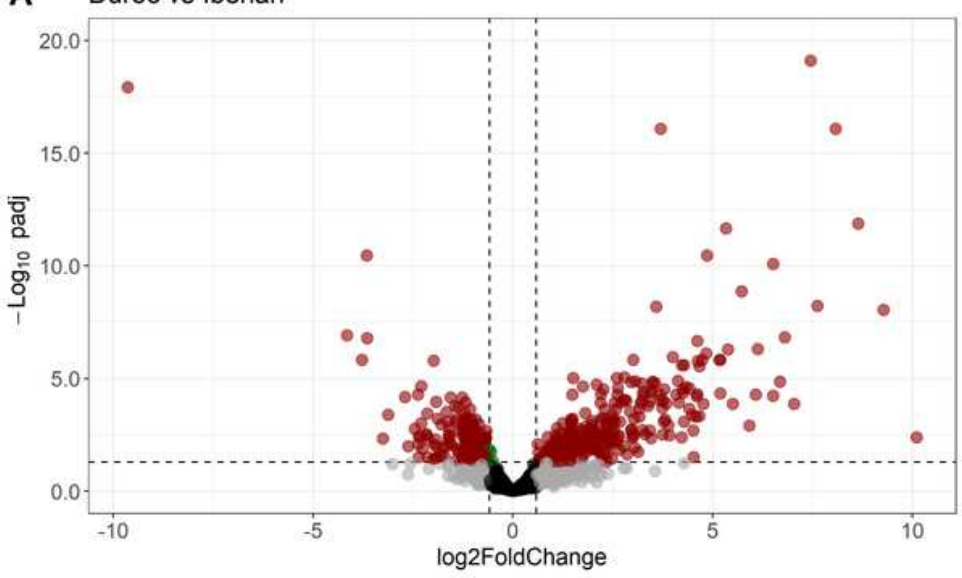

C Duroc vs Iberian

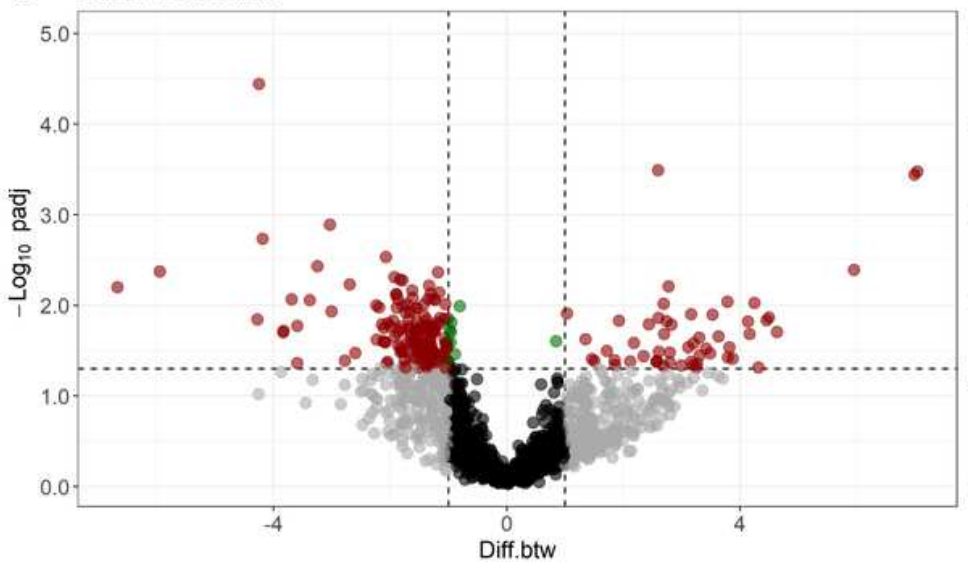

D Control vs High Oleic

B Control vs High Oleic
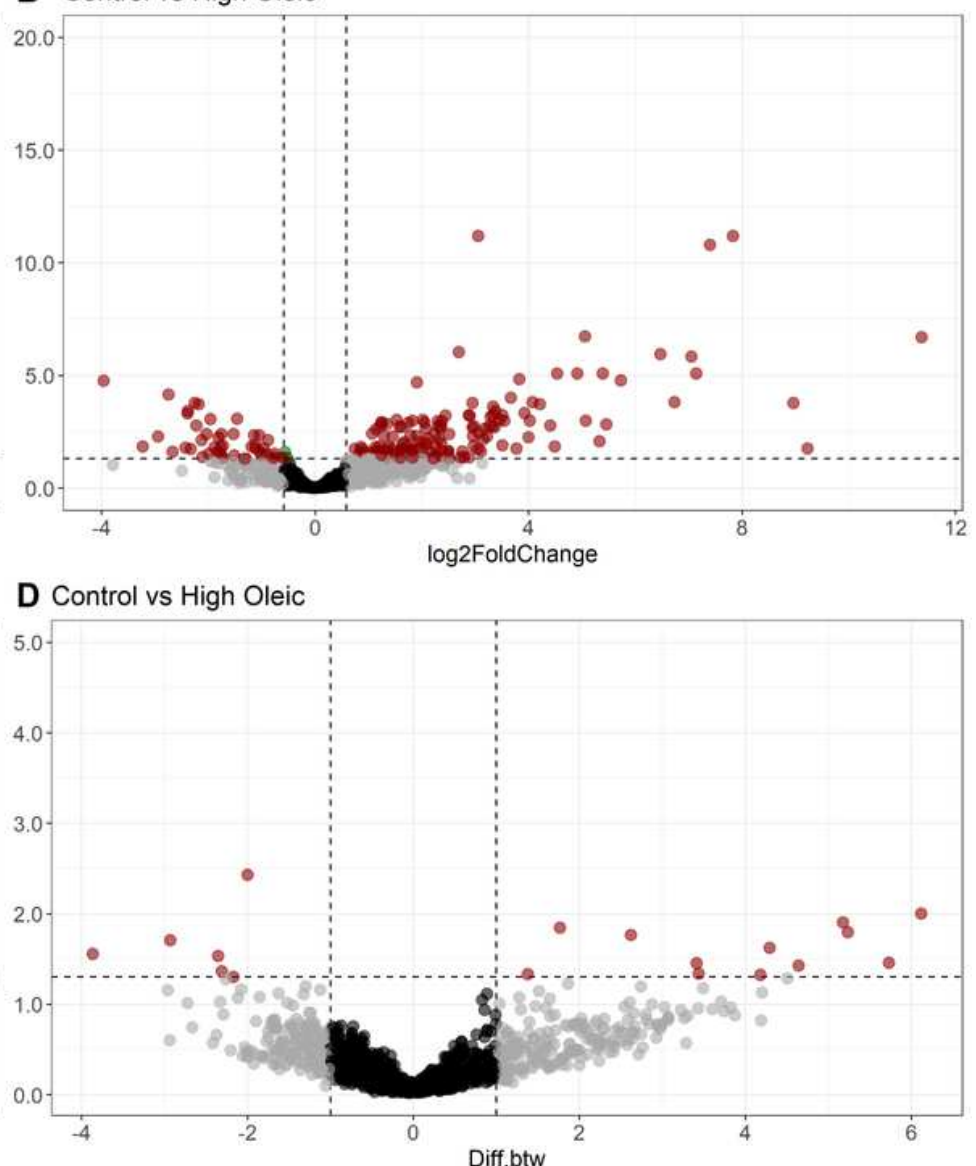

- NS $\log 2 \mathrm{FC} \bullet \mathrm{p}$-val $-\mathrm{p}$-val \& $\log 2 \mathrm{FC}$

\section{Figure 5}

Volcano plots of differentially abundant OTUs using DESeq2 (A, B) and ALDEX2 (C, D), for breed (left) and diet (right) contrasts. Red dots represent OTUs with an adjusted p-value below the FDR cut-off $(0.05)$ and a FC value above 1.5 or below -1.5 for DESeq2 contrasts, or a median difference between CLR values above 1 or below -1 for ALDEx2 contrasts. OTUs with Log2(FC) $<0$ or diff.btw $<0$ are more abundant in Duroc and Control groups, respectively. 


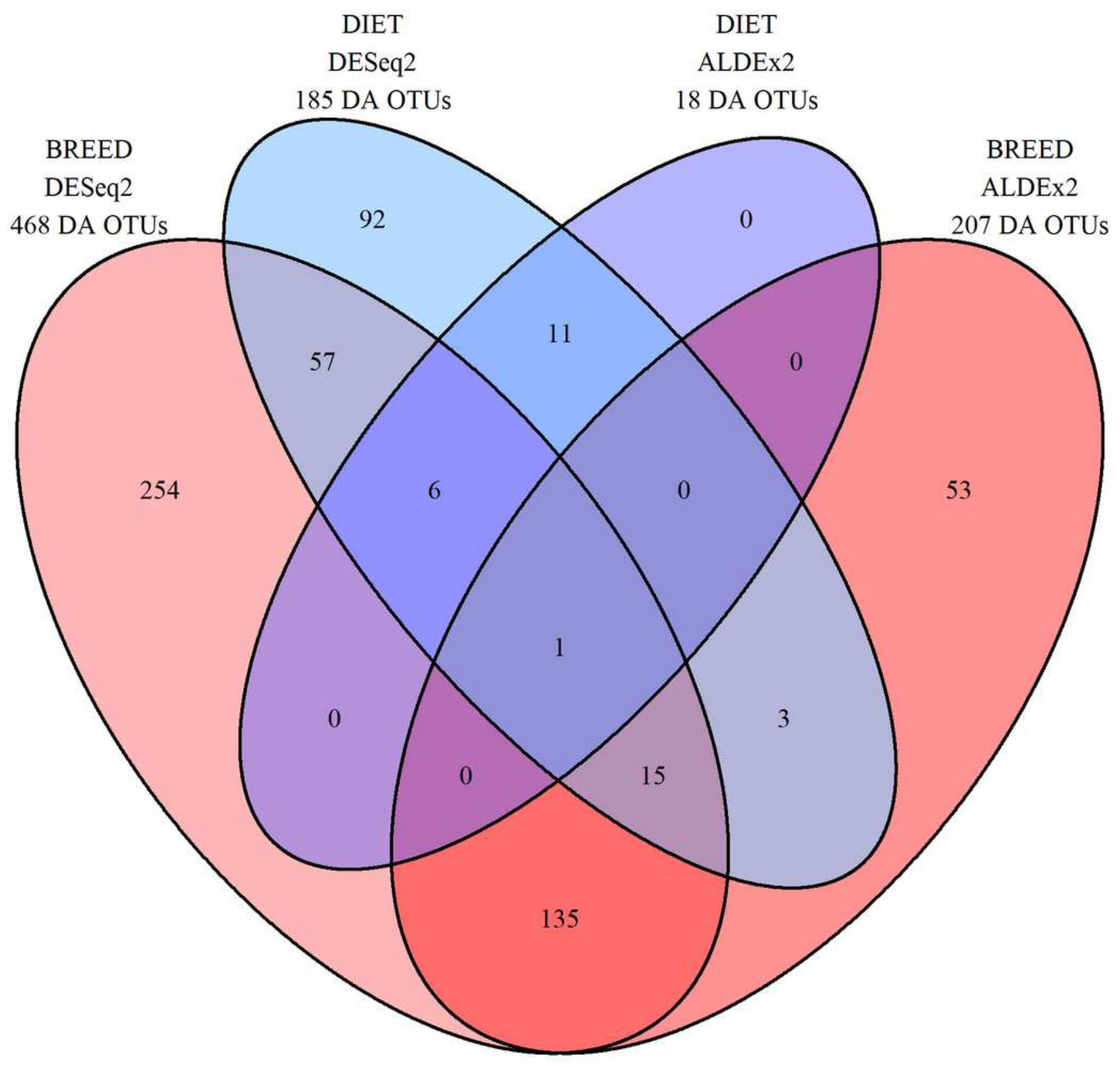

Figure 6

Venn Diagram comparing DA OTUs in Breed (red) and Diet (blue) contrasts using both DESeq2 and ALDEx2. 


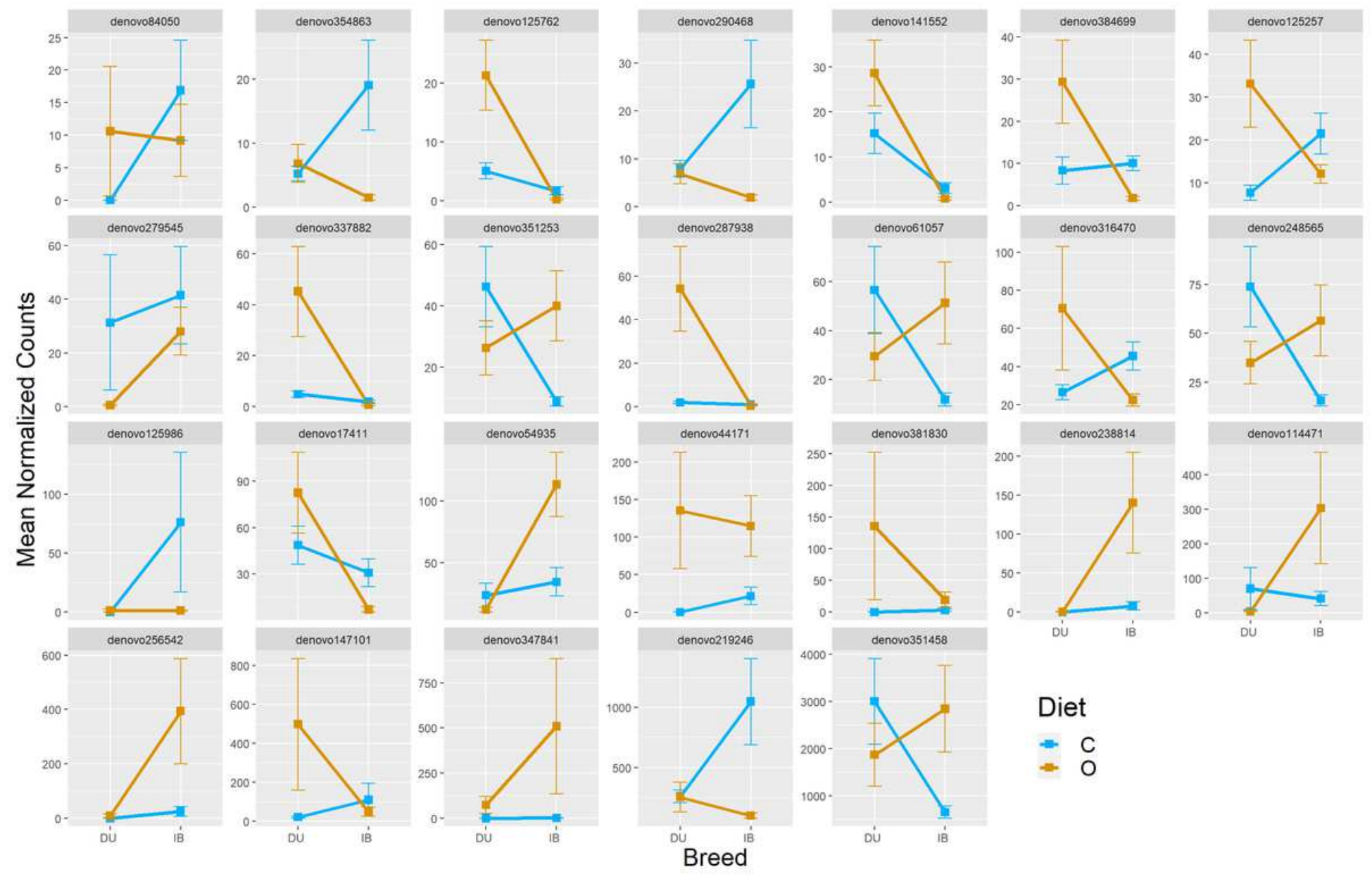

Figure 7

Interaction plots for each OTU with significant interaction between breed and diet factors for DA analysis with DESeq2. Normalized counts from DESeq2 algorithm and standard error of the mean (SEM) are represented in y-axis. 


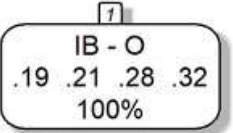

yes Actinobacteria/Epsilonbacteraeota $>=-1.8$ no

$-0$

$\begin{array}{llll}32 & .36 & .25 & .07\end{array}$

$60 \%$

Deferribacteres/Epsilonbacteraeota $>=-2.4$
(4)

DU - C

$.80 .00 \quad .20 \quad .00$ $21 \%$

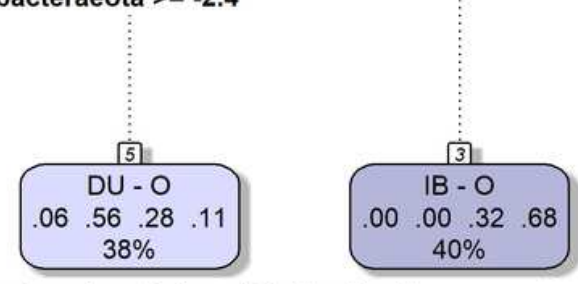

Decision tree: Log-Ratios at Phylum level

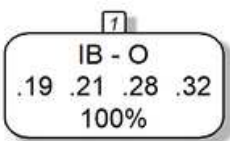

yes Anaerostipes/Corynebacterium_ $1<1.7$ no

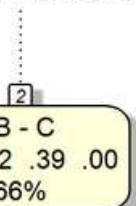

Family_Peptostreptococcaceae/Peptococcus $>=1.7$

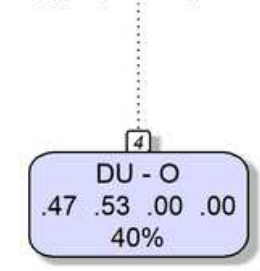

Decision tree: Log-Ratios at Genus level

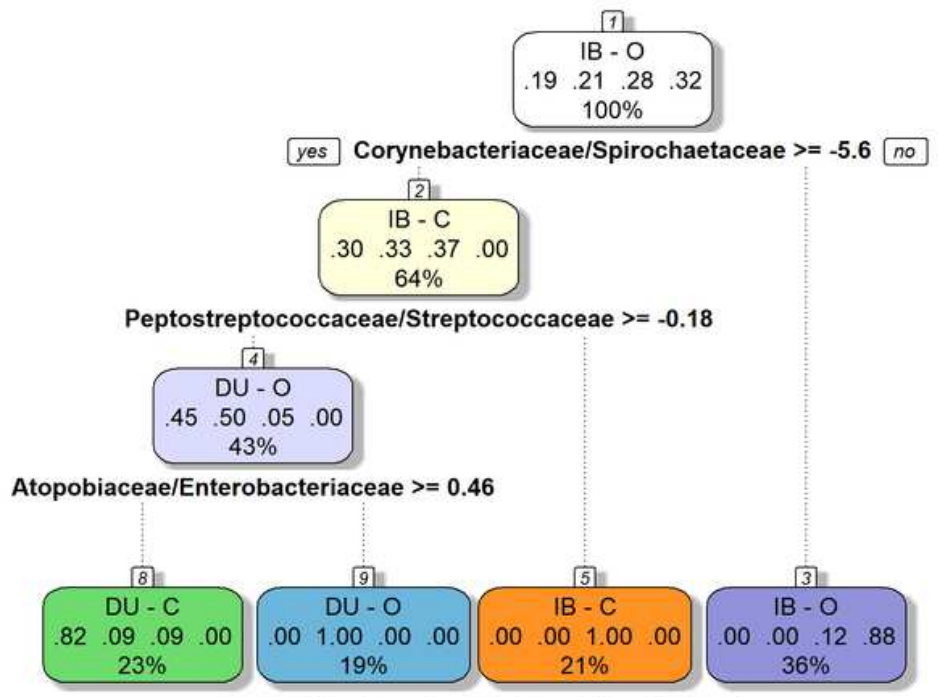

Decision tree: Log-Ratios at Family level

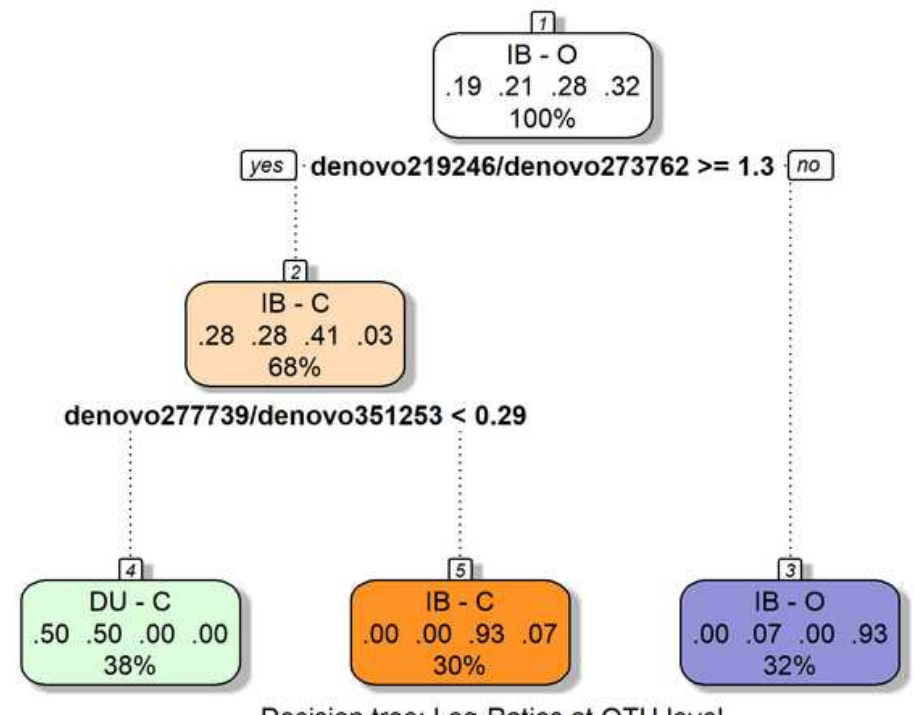

Decision tree: Log-Ratios at OTU level

\section{Figure 8}

RPART trees from pairwise log-ratios at phylum, family, genus and OTU levels. Each decision level indicates the threshold value of one p-LR to classify a sample in one phenotypic group or another. Each node box displays the classification, the probability of each class at that node (i.e. the probability of the class conditioned on the node) and the percentage of observations used at that node. Class probabilities at each node are sorted as: DU-C, DU-O, IB-C and IB-O. 


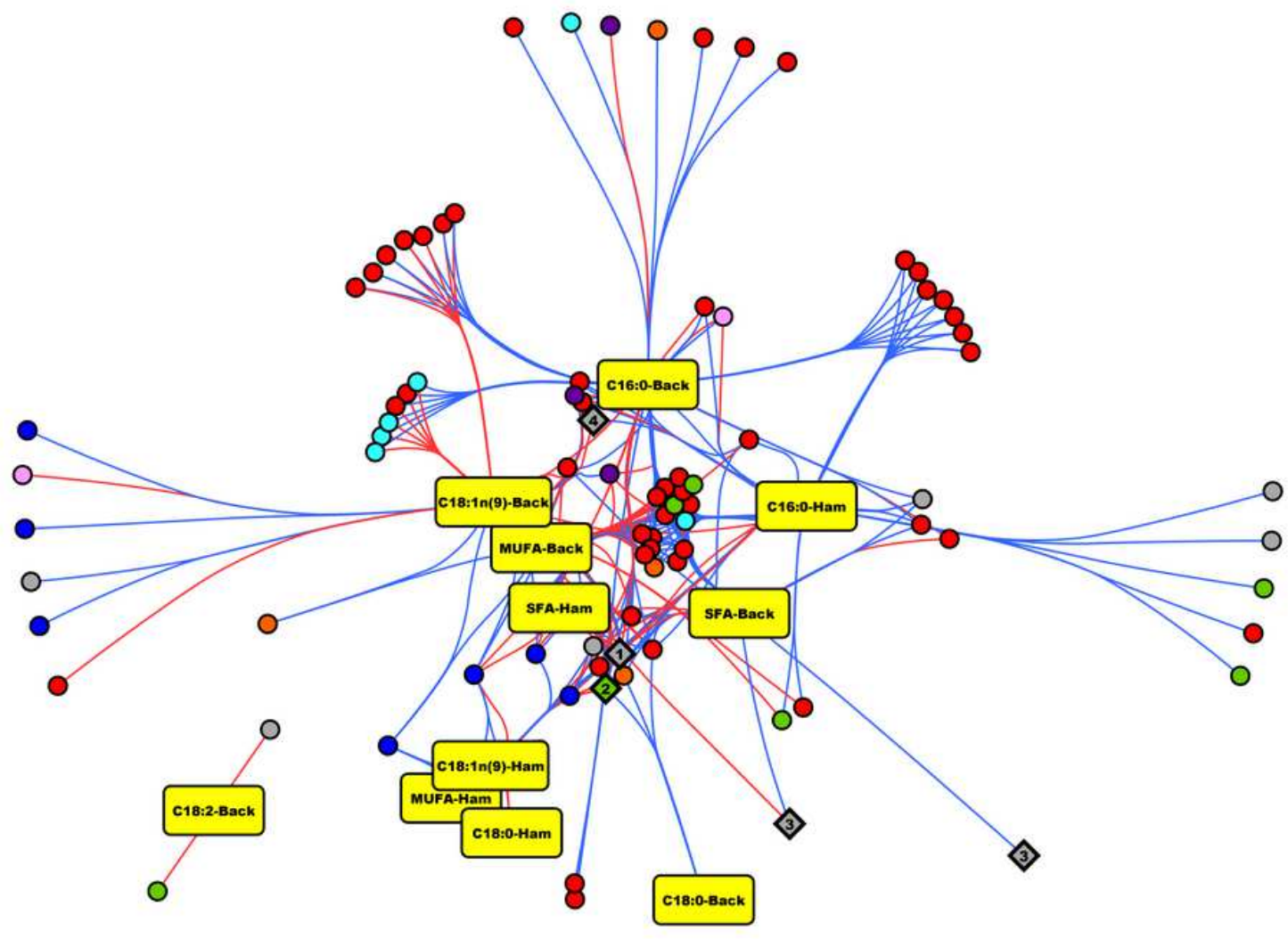

Node Fill Color

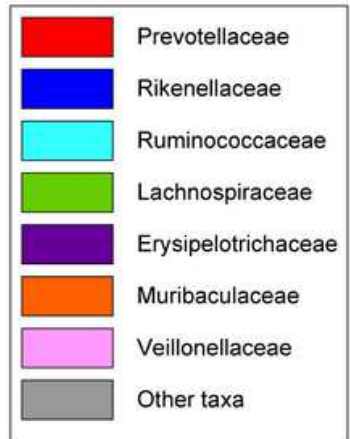

Node Number

\begin{tabular}{|l}
\hline 1) Corynebacterium_1 \\
2 Shuttleworthia \\
3 \\
4. Lactobacillus \\
4. Campylobacter
\end{tabular}

\section{Figure 9}

Network representing individual OTU significant correlation with each phenotypic trait within IB subpopulation, from Limma regression analysis, when no diet correction was applied. Edge colour: red = positive correlation; blue = negative correlation. Central nodes have more connections (i.e., significantly associated to more phenotypic traits) than peripheral nodes. Numbered nodes represent OTUs classified as genera with high relevance due to their abundance patterns in our dataset.

\section{Supplementary Files}

This is a list of supplementary files associated with this preprint. Click to download.

- Additionalfile1.pdf

- Additionalfile2.xlsx

- Additionalfile3.xIsx 